\title{
Temporal and Angular Properties of GRB Jets Emerging from Massive Stars
}

\author{
Brian J. Morsony, Davide Lazzati and Mitchell C. Begelman ${ }^{1}$ \\ JILA, 440 UCB, University of Colorado, Boulder, CO 80309-0440, USA \\ morsony@quixote.colorado.edu; lazzati@colorado.edu; mitchb@jilau1.colorado.edu
}

\begin{abstract}
We study the long-term evolution of relativistic jets in collapsars and examine the effects of viewing angle on the subsequent gamma ray bursts. We carry out a series of high-resolution simulations of a jet propagating through a stellar envelope in $2 \mathrm{D}$ cylindrical coordinates using the FLASH relativistic hydrodynamics module. For the first time, simulations are carried out using an adaptive mesh that allows for a large dynamic range inside the star while still being efficient enough to follow the evolution of the jet long after it breaks out from the star. Our simulations allow us to single out three phases in the jet evolution: a precursor phase in which relativistic material turbulently shed from the head of the jet first emerges from the star, a shocked jet phase where a fully shocked jet of material is emerging, and an unshocked jet phase where the jet consists of a free-streaming, unshocked core surrounded by a thin boundary layer of shocked jet material. The appearance of these phases will be different to observers at different angles. The precursor has a wide opening angle and would be visible far off axis. The shocked phase has a relatively narrow opening angle that is constant in time. During the unshocked jet phase the opening angle increases logarithmically with time. As a consequence, some observers see prolonged dead times of emission even for constant properties of the jet injected in the stellar core. We also present an analytic model that is able to reproduce the overall properties of the jet and its evolution. We finally discuss the observational implications of our results, emphasizing the possible ways to test progenitor models through the effects of jet propagation in the star. In an appendix, we present $1 \mathrm{D}$ and $2 \mathrm{D}$ tests of the FLASH relativistic hydrodynamics module.
\end{abstract}

Subject headings: gamma-rays: bursts — hydrodynamics — shock waves — supernovae: general

\footnotetext{
${ }^{1}$ Department of Astrophysical and Planetary Sciences, University of Colorado at Boulder
} 


\section{Introduction}

The association of gamma-ray bursts (GRBs) with supernova explosions creates an apparent contradiction. On the one hand, the observed gamma-ray spectra require the emitting material to be outflowing at highly relativistic speed (Lithwick \& Sari 2001), limiting its rest mass to a fraction of a solar mass. On the other hand, the jet expands through a dense stellar core, potentially picking up several solar masses in its way. Pioneering work by Woosley and collaborators (MacFadyen \& Woosley 1999; MacFadyen et al. 2001) and by Aloy and collaborators (Aloy et al. 2000) showed that a light jet can reach the surface of a star virtually unpolluted if its head travels sub-relativistically inside the star. In this way, the cold and dense stellar material can be pushed aside. Once the stellar surface is reached, the light jet accelerates and reaches the high Lorentz factors required by $\gamma$-ray observations (see also Matzner 2003 for a simplified analytic treatment).

Once this basic question is answered, two main issues remain open. First, what is the jet launching mechanism at the base of the star and what are the properties of the injected outflow? Second, how does the propagation of the jet influence its properties in the radiative phase, which takes place when the jet has traveled far from its birth site? The first question has been addressed with MHD numerical simulations (Proga et al. 2003; Mizuno et al. 2004; De Villiers et al. 2006; Nagataki et al. 2006). Due to the complexity of dealing with 3D magnetic phenomena in the general relativistic regime, these studies are still in their infancy. This paper deals with the second of these questions. Zhang et al. (2003) performed fixed grid 2D special relativistic simulations of jets in the cores of massive stars. They observed a diverse phenomenology of time dependent properties in the emerging jets, including variations in the opening angle, but their resolution was not large enough to draw robust conclusions at large radii, after the jet breaks out of the star. Lazzati \& Begelman (2005, hereafter LB05) showed, with an analytic model, that the star-jet interaction can be strong and can create observable consequences. They argued that even if a steady jet is injected in the stellar core, its properties at the surface of the star will be strongly time-dependent, so that even the concept of a jet opening angle becomes hard to define in a general sense. They concluded that the time-integrated jet structure would match that of the "Universal Jet" proposed by Rossi et al. (2002). Even though - as we will show in this paper - their approximations were inaccurate in places, and the jet structure they predicted is not observed in the simulations, we confirm the basic concept that the propagation of the jet through the star will strongly influence both, generating strong temporal evolution in their properties. The influence of the jet's initial conditions has been studied recently with a set of 2D simulations (Mizuta et al. 2006). Their jets, however, are cylindrical at the base, different from any previous work and from our simulations presented here, where the outflow is set up to be conical in the core of the star.

In this paper, we present the results of a set of high-resolution 2-dimensional simulations of a jet propagating through a stellar progenitor. The jet is assumed to be already developed and

mildly relativistic at $10^{9} \mathrm{~cm}$ inside the star, even though it is still hot and entropy-rich. The energy release is constant and the engine is left to run for a time longer than the breakout time, i.e., the time at which the jet breaks through the stellar surface. We show that the jet-star interaction 
creates three well-defined phases. The first one is characterized by turbulence. During this phase the jet propagates through and eventually breaks out of the star. The second phase is a transition phase, during which a heavily shocked jet flows through the opened channel. Finally, a more stable configuration is attained, with a freely flowing jet surrounded by a thin boundary layer that progressively widens its opening angle. Some of these phases can be dealt with analytically or semi-analytically with sufficient accuracy, allowing us to understand the origin of the observed behavior. The observational implications of such non-steady flows are numerous, including long dead times during the prompt emission and bumpy, complex afterglows that do not obey the rules of the simple external shock model.

This paper is organized as follows. In $\S 2$ we describe the numerical code used. In $\S 3$ we describe the initial conditions for both the stellar progenitor and the jet in its center while in $\S 4$ we detail our results. In $\S 5$ we develop an analytic modeling of the jet and its propagation, in $\S 6$ we present afterglow calculations based on the numerical results of $\S 4$. We finally summarize our results in $\S 7$.

\section{Numerical Methods}

The simulations presented here were performed using a modified version of the FLASH adaptive mesh refinement (AMR) code (version 2.5) in 2D cylindrical coordinates. The FLASH special relativistic hydrodynamics module utilizes piecewise-parabolic interpolation for computing fluxes at cell interfaces and uses a two-shock Riemann solver for solving the fluid equations. See Mignone et al. (2005) for a full description of the methods used in the relativistic portion of the FLASH code. An adiabatic equation of state (EoS) with a fixed adiabatic index is used. The choice of a single EoS for both the relativistic and non relativistic material is dictated by the need to keep the running times reasonable and by the fact that we will concentrate on the properties of the relativistic material. Future work, that will analyze also the behavior of the star material in detail, will adopt a more realistic EoS. Block refinement is increased if the normalized second derivative of the density or pressure is greater than 0.8 anywhere in the block. Refinement is decreased if the normalized second derivative of the density and pressure is less than 0.2 everywhere in the block. Appendix $\mathrm{A}$ contains results from a number of test problems using this code.

In order to achieve higher resolution near the center of the star and close to the axis of the jet, we have modified the FLASH code so that the maximum level of refinement allowed can be varied over the simulation grid. See $\S 3.2$ for details of the grid setup used for the simulations presented here. In order to improve the efficiency of the simulations, the FLASH code was modified so that quantities in a given cell are only computed and updated if $\bmod \left(n_{\text {step }}, 2^{\left(l_{\text {max }}-l_{i}\right)}\right)=0$, where $n_{\text {step }}$ is the time step number, $l_{\max }$ is the maximum level of refinement anywhere on the grid, and $l_{i}$ is the refinement level of a particular cell. The size of the time step used for computing new values in a cell is correspondingly increased by a factor of $2^{\left(l_{\max }-l_{i}\right)}$. In other words, larger time steps are taken less often for cells at a lower level of refinement. This adjustment is equivalent 
to having a fixed Courant-Friedrichs-Levy (CFL) number at all levels of refinement, rather than a lower CFL number in less resolved cells, as is the default in FLASH. The minimum time step is determined by the maximum velocity anywhere on the grid, ensuring that the CFL condition is never violated. Additionally, in the relativistic case the maximum velocity is always $\sim c(1$ in relativistic units), so the minimum time step is approximately constant. This improvement in efficiency is generally applicable to the entire FLASH code and not just the relativistic portion. Although some operations are still performed on all cells at all time steps, this scheme is always more efficient than the default in FLASH. For the simulations and grid setup described here, this scheme resulted in approximately a factor of 10 decrease in running time. The tests of the adaptive mesh in Appendix $\mathrm{A}$ include this change and do not show any adverse effects.

\section{Setup}

\subsection{Stellar Model}

For the simulations presented here, two different stellar models are used. The first is a realistic stellar model, based on model 16TI from Woosley \& Heger (2006) of a Wolf-Rayet star with an initial mass of $16 \mathrm{M}_{\odot}$, metallicity of $1 \%$ solar and a large angular momentum of $3.3 \times 10^{52} \mathrm{erg} \mathrm{s}^{-1}$. The model has been evolved to core collapse, and with a final mass and radius of $13.95 \mathrm{M}_{\odot}$ and $4.077 \times 10^{10} \mathrm{~cm}$, respectively. (see http://www.ucolick.org/\$ $\backslash$ sim\$alex/GRB2/). Simulations using this model have names beginning with 16TI. The second model is a simple power-law model for a star with a mass of $15 \mathrm{M}_{\odot}$ and a radius of $10^{11} \mathrm{~cm}$. The density profile is modeled as a power law $\rho \propto r^{-2.5}$ and the pressure is set such that $p=p_{0} \rho^{4 / 3}$, where $p_{0}$ is computed based on $p=1.8 \times 10^{18} \mathrm{erg} \mathrm{cm}^{-3}$ at $r=10^{10} \mathrm{~cm}$. This value of pressure at $10^{10} \mathrm{~cm}$ is reasonable based on numerically modeled values for stars of similar size and mass (Heger et al. 2005). Since the stellar pressure is small compared to the jet and cocoon pressure, the actual value of the stellar pressure has little impact on the simulations. All simulations use an ultra-relativistic equation of state with $\Gamma=4 / 3$, so setting $p=p_{0} \rho^{4 / 3}$ ensures that pressure is always small compared to $\rho c^{2}$ inside the star. For both models, the density and pressure exterior to the star are set to $10^{-9} \mathrm{~g} \mathrm{~cm}^{-3}$ and $9 \times 10^{7} \mathrm{erg}^{-3}$, respectively. The mass exterior to the star does not have a significant impact on the dynamics of the simulations presented here, and the exterior density and pressure are set to small, non-zero values for numerical reasons. A CFL number of 0.4 is used for all simulations.

The stars in our simulations are not stable objects since gravity is not included. However, the timescale for the star to expand is large compared to the length of our simulations. After 50 seconds, the end time of our simulations, the material from the edge of the power-law star has moved outward by $8 \times 10^{9} \mathrm{~cm}$, which is smaller than the distance scale for any realistic drop-off in density at the edge of the star. 


\subsection{Grid Setup}

All simulations were run on an identical grid setup. The total grid size is $2.56 \times 10^{11} \mathrm{~cm}$ by $2.56 \times 10^{11} \mathrm{~cm}$. The maximum allowed resolution varies from $7.8125 \times 10^{6} \mathrm{~cm}$ (13 levels of refinement) near the jet input to $2.5 \times 10^{8} \mathrm{~cm}$ ( 8 levels of refinement) far from the coordinate axis outside the star. Figure 1 shows the maximum allowed refinement in different regions. With this setup, the grid has a resolution of $6.25 \times 10^{7} \mathrm{~cm}$ at $10^{11} \mathrm{~cm}$, the surface of the star for the power-law model. This corresponds to an angular resolution of $0.0358^{\circ}$ at this radius. This is a significant improvement over Zhang et al. (2003), who used a grid in spherical coordinates with a resolution of $0.25^{\circ}$, and is comparable to Zhang et al. (2004), who had a resolution of $10^{8} \mathrm{~cm}$ on a cylindrical grid at $10^{11} \mathrm{~cm}$. The variable maximum resolution allows the lower boundary of the grid to be set at $10^{9} \mathrm{~cm}$ above the equator of the star. Zhang et al. (2004) used a fixed grid inside the star, and their lower boundary was placed at $10^{10} \mathrm{~cm}$ above the stellar equator. Zhang et al. (2003) was able to place the lower boundary at a radius of $2 \times 10^{8} \mathrm{~cm}$ in radial coordinates, but at the cost of increasingly poor resolution across the jet at large radii. Similarly, Aloy et al. (2000) could place the inner boundary at $2 \times 10^{7} \mathrm{~cm}$ but the resolution was severely degraded at the star surface.

\subsection{Jet Parameters and Boundary Conditions}

The jet injection is modeled as a boundary condition on the lower edge of the grid at $10^{9} \mathrm{~cm}$. The opening angle and Lorentz factor of the incoming jet are varied between simulations, but are constant at all times in each run. The terminal Lorentz factor, or Lorentz factor at infinity, $\gamma_{\infty}$, is defined as the Lorentz factor that the material would achieve if all its internal energy were converted to kinetic energy. It is calculated as $\gamma_{\infty}=\left(1+4 p / \rho c^{2}\right) \gamma$, where $\gamma$ is the local bulk Lorentz factor. $\gamma_{\infty}$ for the jet material is set to 400 for all simulations and the luminosity of the central engine is set to $5.32 \times 10^{50} \mathrm{erg} \mathrm{s}^{-1}$. Table 1 lists the parameters used for each simulation. Outside the jet injection region, where the boundary values are fixed, the boundary conditions on the lower edge of the grid are reflective, which is appropriate assuming that symmetric jets emerge along both axes of the star. The outer boundaries of the grid use outflow boundary conditions and the symmetry axis is reflecting. Each simulation is run for 50 seconds, giving an energy input of $2.66 \times 10^{52}$ ergs per jet, or a total energy of $5.32 \times 10^{52} \mathrm{ergs}$ assuming symmetric jets. These total energies are comparable to those assumed in previous works (see Zhang et al. 2003 for a discussion).

\section{Results}

The early evolution of each of our models is generally similar to that found in previous simulations (Zhang et al. 2003, 2004). The jet ram pressure generates a bow shock that propagates sub-relativistically through the star. Cold and dense stellar material is pushed to the sides, partly

mixing with shocked jet material to create a hot cocoon that surrounds the jet. This allows the 
younger jet material to propagate relativistically and unpolluted through an evacuated channel along the symmetry axis. Once this material reaches the stellar surface, it accelerates to its final Lorentz factor and can, once it has become optically thin, generate the observed GRB spectra.

Figure 2 shows a time sequence of the evolution of model t10g5. The three upper panels are very similar to figures obtained by other groups with previous simulations, even though our AMR code can capture finer details. The main novelty of our work is shown in the three bottom panels, where the jet is still powered several tens of second after breakout and is evolving inside the star. As we will explain in more detail in the following sections, three phases can be identified. Initially the jet is confined, and hot turbulent material is stored in a cocoon (first two panels). When the jet head reaches the surface, the cocoon is released as a wide angle outflow (third panel). Immediately afterward, a heavily shocked jet flows outside the star (third and fourth panels). Eventually, a more stable configuration emerges (fifth panel) in which the jet is internally free-flowing, and is bounded by a shear layer at the contact discontinuity with the star. Figure 3 shows a snapshot of simulation t10g2 at 30 seconds as an example of the structure present shortly after breakout.

The three phases are identified through the behavior of the on-axis energy flow, as shown in Fig. 4. The transition between the precursor and the shocked jet is defined as the moment at which the energy flow along the axis becomes continuous, albeit variable. The transition between the shocked and unshocked jet is defined as the time at which the on-axis energy flow drops and becomes steady, without prominent variations. Table 2 lists the times at which the cocoon, shocked jet, and unshocked jet reach the initial surface of the star. From these data we can see some expected trends as different parameters are varied. Models with an initial Lorentz factor of 2 (t10g2 and 16TIg2) have longer breakout times than corresponding models with an initial Lorentz factor of 5 (t10g5 and 16TIg5). This is to be expected because a lower Lorentz factor at the same energy implies less momentum in the direction of propagation and a higher pressure (similar conclusions in a different geometry were obtained by Mizuta et al. 2006). This means that the jet will be less tightly collimated and will propagate more slowly.

Model t5g2, with a $5^{\circ}$ injection opening angle, has a slightly earlier cocoon breakout time than the corresponding model with a $10^{\circ}$ opening angle (t10g2). A smaller opening angle means that the same amount of energy and momentum is initially spread over a smaller area, making the jet more penetrating. However, as the jet is being collimated, the initial opening angle is only important at the beginning of the simulation, hence the overall difference between the two simulations is small. The shocked jet breakout time is earlier for model t $10 \mathrm{~g} 2$ by $0.8 \mathrm{~s}$, and the unshocked jet breakout is earlier for model t5g2 by $2 \mathrm{~s}$. These differences appear to depend more on the details of turbulence near the jet head than on the initial opening angle of the jet.

Simulations using the realistic stellar model (16TIg5 and 16TIg2) have earlier breakout times than simulations using the power-law stellar model and identical jet (t10g5 and t10g2, respectively). This is because the realistic stellar model is more compact, giving the star a smaller radius and higher density. The higher density leads to a more tightly collimated jet, which is therefore more 
penetrating. As a result, the jet is able to travel through about the same amount of total mass in a shorter time. It should be noted, however, that the average speed of the bow shock is smaller in the denser, realistic, stellar progenitor.

We also ran a simulation where a wide jet $\left(\theta_{0}=20^{\circ}\right)$ is injected at the center of the star. Model t20g5 appears to be morphologically different from the other simulations. It has the latest cocoon breakout time of any of the simulations, and the jet material along the propagation axis does not reach the surface of the star until the end of the simulation. Before this, relativistic material is deflected around a dense wedge of stellar material and can escape the star at large angles, but it does not appear that this would produce a classical gamma ray burst. This is in part a $2 \mathrm{D}$ effect. In three dimensions, the jet could be deflected sideways to move around the wedge of material, but in our 2D simulations it is forced to be axially symmetric and therefore stalls (see Zhang et al. 2004). Figure 5 compares models t $\log 5$ and $\operatorname{t20g} 5$. The reason why model $\operatorname{t20g} 5$ is different is that the initial opening angle is too large to produce a tightly collimated jet. The pressure of the incoming jet falls as $\theta_{0}^{-2}$, meaning that a wider jet is less penetrating. At 20 degrees, model t20g5 is not penetrating enough to pierce the star in the manner necessary to create a GRB, despite the large energy input of $5.32 \times 10^{52} \mathrm{ergs}$. This simulation may represent a different class of jet-powered supernova or failed gamma ray burst, but it will not be considered in latter portions of this paper that compare our simulations to classical GRB properties. Analogous conclusions were drawn from previous simulations (MacFadyen et al. 2001; Zhang et al. 2003) and for 3D precessing jets (Zhang et al. 2004). It should be remembered, however, that assuming a wide jet at $10^{9} \mathrm{~cm}$ in the star may be unphysical. Even if a wide jet is launched at the black-hole scale, recollimation is likely to take place at radii $10^{8}-10^{9} \mathrm{~cm}$ (see model JA in Zhang et al. 2003). As a consequence, an initial condition with a narrow, entropy rich, jet (model t5g2) is more realistic than a wide fast outflow (model t20g5) at the inner boundary of our simulations.

\subsection{Angular Distribution of Energy}

A snapshot file containing all the simulation data is produced every $1 / 15$ th of a second in simulation time. The energy flux is determined as a function of angle and time by taking a simulation snapshot and finding all the points that will cross a fixed radius in the next $1 / 15$ th of a second. The energy at each point is spread equally over an angle of $\pm 1 / \gamma_{\infty}$ from the direction of motion of the fluid at that point. This approximately accounts for the hydrodynamic spreading (or sideways expansion) of that element of the jet and for the relativistic beaming of radiation eventually emitted by that material. The energy is then placed into angular bins. The total energy in each angular bin can then be calculated by adding the energy from all points, and correcting for geometrical effects to give the energy flux in each bin. The energy in material above a given terminal Lorentz factor (Lorentz factor at infinity) can be found by excluding points with material below that $\gamma_{\infty}$. The results from each snapshot can be added over time to find the total energy seen at a fixed radius at different angles for the entire length of the simulation, or for shorter 
time intervals. With this data, we can examine the amount of energy crossing a fixed radius as a function of time, angle, and minimum Lorentz factor. Bear in mind that, given the definition of the angle adopted, the energy distribution may be modified by hydrodynamic interactions during the propagation to larger radi] 1 . For the results presented here, data from our simulations have been taken at $2.4 \times 10^{11} \mathrm{~cm}$ and placed into 45 angular bins arranged in the following manner: 14 bins spaced every $0.25^{\circ}$ with centers ranging from $0.125^{\circ}$ to $3.375^{\circ}, 17$ bins spaced every $1.0^{\circ}$ with centers ranging from $4.0^{\circ}$ to $20.0^{\circ}$, and 14 bins spaced every $5.0^{\circ}$ with centers ranging from $23.0^{\circ}$ to $88.0^{\circ}$.

\subsubsection{Energy vs. Angle}

In order to determine the observational characteristics of a gamma ray burst produced from our simulations, we examine the amount of energy directed toward observers at different angles. Figure [6] shows the total energy crossing a radius of $2.4 \times 10^{11} \mathrm{~cm}$ during the simulation. Minimum terminal Lorentz factors of 1.01, 2, 10, 50, and 200 have been used for the different lines in the figure. The energy has been converted to units of isotropic equivalent energy available at different angles. The observed isotropic energy would then be the available energy times the efficiency of converting this energy to gamma rays. Different minimum Lorentz factors are presented because the efficiency of gamma ray production is likely to vary with Lorentz factor.

For each simulation, the total isotropic equivalent energy $d E / d \Omega$ is constant or slowly decreasing from the jet axis out to some cutoff. Inside the cutoff, energy goes as $\sim$ constant to $\theta^{-1}$. Beyond this, the energy decreases rapidly. In some cases there is a significant contribution from precursor energy at large angle, but when this is removed (Fig. 7), the cutoff in energy becomes even sharper. For large $\gamma_{\infty}$, this decrease in energy can be fit by an exponential or a steep power law $\left(d E / d \Omega \sim \theta^{-4}\right.$ or more). If low-energy particles are included $\left(1.01 \leq \gamma_{\infty} \leq 2\right)$ then energy falls off as $\sim \theta^{-2}$ at large angles. These particles should not contribute to the prompt burst, as they do not have a Lorentz factor large enough to produce gamma rays, but they can produce X-rays and can be important to the afterglow energetics. This differs from the Zhang et al. (2004) finding that the energy is distributed as $d E / d \Omega \sim \theta^{-3}$. The difference is due to a combination of several factors. First, since their simulations are performed on a fixed grid, the resolution is poorer at the stellar surface compared to our AMR simulations. Second, Zhang et al. (2004) consider a small range of angles for their $\theta^{-3}$ fit $\left(3^{\circ}<\theta<15^{\circ}\right)$. As a matter of fact, some of our models (especially 16TIg2 and t10g5) could be fit by $\theta^{-3}$ power-laws in small angle intervals. Finally, their simulation domain inside the star is only from $10^{10} \mathrm{~cm}$ to $8.8 \times 10^{10} \mathrm{~cm}$, a volume that may be too small to allow the development of turbulence that would result in mixing of the jet material with the stellar

\footnotetext{
${ }^{1}$ An alternative definition of the angle is provided by the positional angle of the point with respect to the origin of the coordinates. This definition is also prone to change with the expansion of the flow if the velocity and position vectors are not aligned.
} 
material. From our simulations and the comparison with previous work, it can be concluded that the energy distribution with angle is not a robust property of the collapsar, but depends on the stellar structure and the jet injection parameters.

As discussed above, the passage of jet material at a fixed radius consists of two phases: the passage of the shocked and unshocked jet. Figure 8 shows the total energy from each of these phases in model 16TIg5 for $\gamma_{\infty} \geq 50$ at a radius of $1.2 \times 10^{11} \mathrm{~cm}$. The shocked phase of the jet has an approximately uniform energy distribution with a sharp cutoff at the edge of the jet. During the unshocked phase, however, mass and energy are concentrated in the shocked boundary layer that surrounds the unshocked core of the jet. This means the unshocked jet does not contribute much energy near the jet axis. However, at angles outside where the shocked phase of the jet is visible, the unshocked phase is the dominant energy source. In addition, while the energy in the shocked jet phase is fixed by the stellar properties, the unshocked jet can extend in time and become more and more prominent. Only self-consistent simulations of the jet feeding, launching, and propagation can pin down any absolute normalization between the energies of the two phases. At any angle, the unshocked phase only contributes significant energy while the thin shocked boundary layer

covers that angle. As the opening angle of the jet is increasing during this phase, an observer will only see the boundary layer for a relatively short time. Increasing the duration of energy injection will therefore increase the final opening angle of the jet, but it will not significantly increase the isotropic equivalent energy seen by observers already within the jet.

\subsubsection{Phases of Jet Evolution}

Figure 9 shows logarithmic energy (brightness) contours as a function of time (vertical axis) and angle (horizontal axis) for model 16TIg5. Different panels show different minimum $\gamma_{\infty}$ cutoffs. The figure shows that there are three phases in the energy flux seen at a fixed radius. First there is a precursor phase, preceding significant on-axis energy flux, followed by a shocked jet phase, and an unshocked jet phase.

The precursor phase can be divided in two subsequent events. First a thin, nearly isotropic shell of mildly relativistic material, visible in the lower energy bands of Fig. 9. This phase is associated to the shock preceding the jet expanding into a density gradient and carries very little energy. For this reason it has observational consequences only if the other phases of the GRB are not visible due to viewing angle constraints (see $\S$ 4.2.4). This initial precursor is followed by a mixture of jet and stellar material which is peaked at several degrees off axis with less energy on axis. This is the material that accumulates around the jet in the form of a cocoon (Ramirez-Ruiz et al. 2002) during the initial phases when the jet is confined inside the star. This material generally has a lower Lorentz factor than the jet and is most clearly visible in the lower energy plots. A further discussion of this material is given in $\S 4.2$,

After the cocoon material, the shocked jet emerges. Differently from the cocoon material, the 
shocked jet has a large Lorentz factor at the stellar surface. During the shocked phase, jet material between the jet head and reverse shock is passing by. The shocked portion of the jet has a highly structured interior, including turbulent structures and internal shocks. Although this makes the energy flux and width of the jet variable during this period, there is no clear trend with time in the jet properties.

Finally, the jet settles into a quasi-stationary configuration, which we call the unshocked phase. The reverse shock has passed the radius we are looking at, and the jet consists of unshocked material surrounded by a narrow boundary layer of shocked material. During this phase the jet does not have the variable structure seen in the precursor and shocked phases, but the width of the jet clearly increases with time (see $\S$ 4.1.3). Figure 10 shows the Lorentz factor and pressure along the jet axis of model 16TIg5 at the time at which the shocked-unshocked jet boundary crosses the star surface. The figure confirms that the core of the unshocked jet, inside the boundary layer, is free streaming. Dashed lines show how pressure and Lorentz factor behave in a free-streaming, pressure dominated jet; the simulation result is in excellent agreement with this behavior. Note that the Lorentz factor deviates from the asymptotic solution at large $\gamma$. This is due to the fact that at such highly relativistic speeds, the pressure-dominated approximation does not hold any more. The comparison of Fig. 10 with previous results (e.g. Fig. 2 in Aloy et al. 2000, Fig. 4, 5, and 6 in Zhang et al. 2003, or Fig. 6 and 7 in Mizuta et al. 2006) reveals much sharper features in our results. This is likely due to the increased resolution of the AMR code with respect to fixed grid codes.

It is interesting to speculate on the temporal properties of the three phases. It is usually assumed that the light curve of the GRB prompt emission is due to internal shocks driven by Lorentz factor inhomogeneities in the flow. These inhomogeneities are supposed to be imprinted by the central engine. The propagation of the outflow through the star, however, is likely to modify these structures, erasing some and amplifying others. Which ones are erased and which amplified will depend on the phase of the jet evolution. The precursor material is made of jet and stellar material that has been completely reshuffled and shocked in the bow shock and turbulent eddies surrounding the jets. As a consequence, the ejection history of the central engine has been forgotten. A similar conclusion holds for the shocked jet, even though some trace of the engine properties may be retained. In the unshocked jet, however, and especially in its freely expanding

core, all variability imprinted by the engine will be frozen and advected to the radiative phase. The properties of the inner engine will therefore be more clearly seen in the tail of the GRB emission. Unluckily, this is the faintest phase.

\subsubsection{Opening Angle}

As noted from previous simulations (Zhang et al. 2003) and discussed theoretically in LB05, the opening angle is not a constant property of the outflow emerging from the star, nor is it an easily measurable quantity. We define the opening angle as follows. We first select all points that 
will cross a fixed radius within $1 / 15$ th of a second (the same points used to find energy flux) and with a minimum value of $\gamma_{\infty}$. We then compute the angles associated with all these points and define the jet opening angle as the largest. In this case, we define the angle as the geometrical angle of the point with respect to the origin and polar axis of the coordinates, since adopting the velocity vector angle introduces substantial noise (see footnote 1).

Figure 11 shows opening angle vs. time for different minimum values of the terminal Lorentz factor $\left(\gamma_{\infty}\right)$ at a radius of $1.2 \times 10^{11} \mathrm{~cm}$ in our simulations. This figure emphasizes again the three phases of jet evolution. As material first reaches this radius, the opening angle is very wide for all but the highest values of $\gamma_{\infty}$. This is due to the precursor material discussed in $\S 4.2$. Following this is the shocked portion of the jet, characterized by a fairly constant opening angle and no consistent evolution with time. Using different values of $\gamma_{\infty}$ gives widely different opening angles, ranging, for example, from $\sim 2^{\circ}$ for $\gamma_{\infty}=200$ to $\sim 8^{\circ}$ for $\gamma_{\infty}=2$ in model 16TIg5. This is because material at the edge of the jet is partially mixed with stellar material, lowering its terminal Lorentz factor. After the shocked portion of the jet has passed, the jet consists of an unshocked core with a shocked boundary layer along the edge. As the unshocked jet passes, the opening angle of the jet is slowly but consistently increasing. The rate of increase is always less than linear and can be well fit by a logarithmic increase. The rate of increase is obviously much slower than the exponential increase predicted in LB05 but is in reasonable agreement with the semi-analytic results presented in this paper $(\S$ (5). The thickness of the boundary layer, measured as the difference between the opening angles for $\gamma_{\min }=2$ and $\gamma_{\min }=200$, decreases with time. For model 16TIg5, the thickness goes from $\sim 6^{\circ}$ in the shocked phase down to $\sim 4^{\circ}$ by the end of the simulation (see Fig. 11).

Although the intrinsic opening angle of the jet is as small as $\theta_{0}=5^{\circ}$, at late times the jet will be over-pressured at the base and expand to a larger opening angle. After cocoon breakout, the pressure in the cocoon decreases exponentially, while the incoming jet pressure remains fixed. When the cocoon pressure drops below the incoming jet pressure, the jet will expand near its base by an angle of up to $1 / \gamma_{0}$, where $\gamma_{0}$ is the Lorentz factor of the incoming jet. For $\gamma_{0}=5$ this is about $11^{\circ}$. Therefore, a jet with $\theta_{0}=5^{\circ}$ and $\gamma_{0}=5$ can have a maximum opening angle of about $16^{\circ}$. Pressure in the unshocked jet drops as $r^{-4}$, so at large radii the jet is still being collimated by the cocoon pressure. In all simulations presented here, the opening angle of the jet at large radii is always less than the maximum opening angle for that simulation.

\subsection{Precursors}

As the jet propagates through the dense material of the star, a high density wedge of stellar material develops at the head of the jet. The jet material does not penetrate this wedge, but instead moves to the sides. Eventually this material will curl back on itself, creating large vortexes in advance of the narrowly collimated jet (Fig. 2). As the jet continues to propagate, the vortexes will eventually detach from the jet and be swept backward, relative to jet propagation, in a phenomenon

known as vortex shedding (Scheck et al. 2002; Mizuta et al. 2004). New vortexes will then develop 
at the head of the jet in a repeating cycle. However, when the cocoon of material surrounding the jet breaks out of the star and into the low density material surrounding it, the cocoon material is released and these vortexes are no longer swept backward. Whatever material is being shed from the head of the jet at this time is then free to expand ballistically. The result is a significant amount of relativistic material escaping over a large angle at close to the breakout time. Even though the details of the process require a 3D simulation for a deep investigation, the formation of a cocoon of high pressure material is unavoidable (see Ramirez-Ruiz et al. 2002 for discussion of cocoon properties). Because the opening angle of the jet increases slowly, for off-axis observers there can be a long delay between when the cocoon material is seen and when the jet is seen. This vortex material could therefore be responsible for precursor events seen 10s of seconds before the main gamma ray burst (Lazzati 2005; Lazzati et al. 2005). In our simulations, lasting 50 seconds, delays of up to $\sim 20$ seconds were seen between the precursor material and jet material at certain angles (Fig. 12). At larger angles, the jet never comes into view, but the precursor is still visible. This could account for the observed soft, low-energy gamma ray bursts such as GRB980425 (associated with SN 1998bw, Galama et al. 1998). Whether this faint GRB had a relativistic jet associated with it is still a matter of lively debate (Waxman 2004).

\subsubsection{Precursor Energetics}

To estimate the total energy contained in the precursor, we first find the isotropic energy vs. angle of the precursor at $2.4 \times 10^{11} \mathrm{~cm}$, as described above ( $\$ 4.1$ ). This energy can then be added over angle to give the total energy in the precursor. The mode and full width at one tenth of the maximum of the energy-weighted direction of motion can also be calculated to give an idea of the angle over which the precursor is visible. All those quantities are given in Tab. 3 for $\gamma_{\infty}>10$ and $\gamma_{\infty}>50$.

For the five simulations, the total precursor energy with $\gamma_{\infty} \geq 10$ ranges from about $3 \times 10^{50}$ to $5 \times 10^{51} \mathrm{ergs}$, with $45 \%$ to $75 \%$ of that energy carried by material with $\gamma_{\infty} \geq 50$. This means that $\gtrsim 1 \%$ of the total input energy of our simulation, $2.66 \times 10^{52}$ ergs per jet, ends up as relativistic material in the precursors. This material is spread over a wide opening angle $\left(\sim 40^{\circ}\right)$, so the isotropic equivalent energy of the precursor emission should be within an order of magnitude of the total precursor energy. Models with a later breakout time typically have more energy in the precursor material, which is expected since a longer propagation time allows more relativistic material to be shed from the jet and because simulations with a lower initial Lorentz factor have later breakout times and have less momentum in the jet, making it easier to deflect. 


\subsubsection{Precursor Energy vs. Angle}

Figure 13 shows isotropic equivalent energy of the precursor vs. angle for our simulations. The isotropic equivalent precursor energy can be up to $2 \times 10^{52}$ ergs, $\sim 2 \%$ of the $10^{54}$ ergs typically seen in the core of the jet. Note in Fig. 13 that near the axis there is usually less precursor emission. This is because vortex material is being deflected away from the head of the jet. This is in part a $2 \mathrm{D}$ effect. In $3 \mathrm{D}$, the jet would be able to wobble and the precursor material would not have to be rotationally symmetric. This should increase the amount of precursor material on axis. However, the qualitative effects would be the same. Material would still be shed from the head of the jet, creating a region of relativistic material spread over a wide opening angle near the head of the jet.

The precursor energies found in our simulations are sufficient to account for observed precursor emissions, which typically produce $\sim 1 / 1000$ th as many photons as the complete GRB (Lazzati 2005). As shown in Fig. 13, the isotropic equivalent precursor energy typically peaks at $10^{52}$ ergs. The absolute energies of observed precursors are not known because the redshifts of GRBs with precursors have not been measured. However, it is possible that precursors are only detected in relatively nearby bursts, which would on average be intrinsically fainter than GRBs in general. This could further reduce the amount of energy needed to produce the observed precursor emission, in the case that precursors have less energy available than our simulations show or are less efficient at producing gamma rays.

\subsubsection{Precursor Lorentz Factors}

Table 3 also contains the ratios of precursor energies for material with $\gamma_{\infty} \geq 50$ to $\gamma_{\infty} \geq 10$. As highly relativistic material $(\gamma \sim 100)$ is needed to produce high-energy gamma rays, this ratio

should reflect the spectral hardness of gamma ray emission from the precursor material. The precursor material is less relativistic than the jet material because some stellar material has been mixed into it. Whereas the jet material typically has $\gtrsim 80 \%$ of its energy in material with $\gamma_{\infty} \geq 50$, the precursor material has $\sim 60 \%$ of its energy in material with $\gamma_{\infty} \geq 50$. A larger percentage of energy in mildly relativistic material could produce a softer spectrum of emitted radiation, as observed in GRB precursors (Lazzati 2005) and low-energy GRBs (Galama et al. 1998).

\subsubsection{Isotropic Precursors}

When the cocoon breaks out of the star, before the cocoon energy of is released, there is a thin shell of mildly relativistic material formed at the leading edge of the expanding material. This shell provides a second possible source of precursor emission. The shell is nearly isotropic and is visible in Fig. 9, Note that this shell is not well resolved in the simulations presented here. The isotropic equivalent energy in this shell is $\sim 10^{47}$ to $\sim 10^{49} \mathrm{ergs}$, far lower than the energy of a typical GRB. 
It is therefore unlikely that radiation from this material would be detected along with a classical GRB. The shell material also has a low terminal Lorentz factor, typically around 5, and therefore may not be able to produce gamma ray photons. However, this material could still produce X-ray photons and may be observed as an X-ray flash by observers far off-axis, who see emission from neither the jet nor the vortex precursor material.

Between the jet material, vortex material and isotropic shell material, observers at different angles could see the same event as a classical GRB without a precursor, a classical GRB with a precursor, a low-energy GRB, or an X-ray flash. Figure 12 shows plots of energy flux vs. time for 4 different angles to illustrate different types of observed events.

At a viewing angle of $1.125^{\circ}$, a large energy flux begins to arrive about 15 seconds after the start of the simulation and last for 18 seconds. After this there is a small amount of energy still arriving (from the unshocked jet) until the end of the simulation. At $5^{\circ}$, the precursor is visible as a spike of emission beginning at $\sim 14$ s and lasting for about 1 s. This is followed by $\sim 15$ seconds with low energy flux. Emission then begins again as the edge jet comes into view. After 44s the emission reaches a lower constant level as the unshocked portion of the jet comes into view. At $7^{\circ}$, the precursor is again visible starting at $14 \mathrm{~s}$, but the jet does not come into view until $\sim 20$ seconds later. At $12^{\circ}$, the precursor is still seen at $14 \mathrm{~s}$, but the jet is never visible.

\subsection{Effects of Resolution}

In order to test the effects of resolution on our simulations, we have carried out a version of $16 \mathrm{TIg} 5$ at half the resolution of our other simulations. In the low-resolution version of model 16TIg5, there is not a distinct precursor phase preceding the shocked jet, as can be seen in Fig. 14. This appears to be because the resolution of this model is not sufficient for vorticity to develop at the head of the jet. The thin isotropic shell is, however, seen in the low resolution model. In the first three seconds after reaching $2.4 \times 10^{11} \mathrm{~cm}$, the jet is wider at lower energies than at later times and is narrower at high energies. Although this structure is not a separate precursor as seen in the high resolution simulations, it could still give rise to precursor emissions seen off-axis before the jet comes into view.

Despite a lack of distinct vortex structures, the evolution of the shocked and unshocked phases are very similar to the high resolution model. Because material at the head of the jet has not been deflected, the shocked portion of the jet is larger in the low resolution model. In other words, the reverse shock has propagated farther into the jet at $2.4 \times 10^{11} \mathrm{~cm}$ in the low resolution model. This would be expected as material is not being shed from the jet as in the high resolution model.

The breakout times of the cocoon and shocked jet are about 3 seconds earlier in the low resolution simulation, and unshocked jets have nearly identical breakout times (Tab. 2). Figure 15 compares the total energy vs. angle at the two resolution and shows that they are very similar. Figure 16 compares the opening angle vs. time of the two resolutions. The low resolution plot 
shows a wider opening angle, particularly at the lower Lorentz factor cutoffs, but this is expected due to the lower resolution. Other than this, the time evolution of the opening angle is nearly identical in the two simulations.

\section{Analytic Modeling and Interpretation}

More insight into the results of the numerical work can be achieved if the dominant processes that shape the jet evolution and its interaction with the star can be singled out. To this aim, we developed an analytical description of the jet-cocoon-star interaction. We find that the phase during which the jet is confined inside the star (before the breakout) and the unshocked jet phase can be reasonably well approximated with a semi-analytic treatment. This allows us to compute breakout times, precursor energetics, the amount of energy given to the star and the late time evolution of the jet properties.

In LB05 we explored the dynamics of the jet-cocoon interaction under the monolithic jet assumption, in which the jet is assumed to be uniform across its section. In addition, the jet is assumed to satisfy Bernoulli conditions, i.e., no significant dissipation by shocks. As a consequence, the pressure exerted by the jet on the cocoon material is only the internal pressure (which is relativistically invariant for a perpendicular Lorentz boost),

$$
p_{j}=\frac{L_{j}}{4 \Sigma_{j} c \gamma_{j}^{2}},
$$

where $L_{j}$ is the jet luminosity, $\Sigma_{j}$ is the jet cross section and $\gamma_{j}$ is the jet Lorentz factor. Under this approximation, LB05 found that the jet reaches the stellar surface very narrow and spreads exponentially afterwards.

The FLASH numerical simulations we present show that the jet does not have a uniform distribution of pressure and density but rather develops a boundary layer structure (see Fig. 22). The core of the jet is freely streaming out to the point at which it collides with the boundary layer, which in turn flows parallel to the jet-cocoon boundary. In addition to the jet internal pressure, we have therefore the ram pressure due to the deflection of the free streaming jet by the boundary layer. Figure 17] shows a sketch of the jet geometry that we consider.

Simple geometry allows us to derive the pressure balance equation

$$
p_{\text {cocoon }}=p_{j}+4 p_{j} \gamma_{j}^{2} \sin \left[\operatorname{atan}\left(\frac{d z}{d r_{\perp}}\right)-\operatorname{atan}\left(\frac{z}{r_{\perp}}\right)\right],
$$

where $z$ is the coordinate along the jet and $r_{\perp}$ is the perpendicular size of the jet. Eq. 2 can be simplified in the approximation of a narrow $\left(z \gg r_{\perp}\right)$ relativistic jet (for which the ram pressure is much larger than the internal pressure) yielding a differential equation of the form

$$
\frac{d r_{\perp}}{d z}=\frac{r_{\perp}}{z}-r_{\perp}^{2} K
$$


where $K=\pi c p_{\text {cocoon }} / L_{j}$ is a constant related to the ratio of the ram to cocoon pressures. Eq. 3 has an analytic solution of the form

$$
r_{\perp}=\frac{2 z}{K z^{2}+C}
$$

where $C$ is a constant of integration. Eq. 4 can be rewritten in a clearer form as

$$
\theta_{j}=\frac{2 \theta_{0}}{2+K \theta_{0}\left(z^{2}-z_{0}^{2}\right)} .
$$

In this form is easy to see how the opening angle of the jet is initially constant, but decreases as the jet propagates. For very large values of $z$ the jet tends to close on itself. This equation will be used in the following to study the time dependence of the jet opening angle. We now concentrate on the parameter $K$. The missing piece of information to derive it is the cocoon pressure.

To compute the cocoon pressure we develop the approximations of Begelman \& Cioffi (1989) and the jet propagation description of Matzner (2003). The cocoon evolution is governed by the first principle of thermodynamics which, for a relativistic temperature cocoon, reads

$$
d \rho_{\text {cocoon }}=\frac{d Q-4 / 3 \rho_{\text {cocoon }} d V_{\text {cocoon }}}{V_{\text {cocoon }}}
$$

where the evolution of the cocoon volume is computed as

$$
\frac{d V_{\text {cocoon }}}{d t}=2 \pi \int_{z_{0}}^{z_{h}} r_{\text {cocoon }, \perp} v_{\mathrm{sh}} d z .
$$

Here $v_{\text {sh }}=\sqrt{\rho_{\text {cocoon }} / 3 \rho_{\star}}$ is the cocoon expansion velocity (Begelman \& Cioffi 1989; $\rho_{\star}$ is the matter density of the star), $r_{\text {cocoon }, \perp}$ is the transverse size of the cocoon, $z_{0}$ is the location of the base of the jet and $z_{h}$ is the position of the head of the jet.

The energy input into the cocoon, $d Q$, is calculated differently in the two phases: when the whole jet is inside the star (the cocoon is bounded) and when the jet has broken out (the cocoon is unbounded). In the first phase, the energy input is from the dissipation of the jet energy. In the second phase, energy is lost through a channel at the stellar surface, that we assume to have a surface area equal to half the jet cross section 2 . We obtain

$$
d Q=\left\{\begin{array}{cc}
L_{j}\left(1-\beta_{h}\right) & \text { jet in star } \\
-\rho_{\text {cocoon }} \Sigma_{j} / 2 c_{s} d t & \text { jet outside the star }
\end{array},\right.
$$

where $\beta_{h}$ is the speed of the head of the jet in units of $c$.

Equations 1, 2, 6, 7, and 8 constitute a solvable system of equations. To check the validity of the assumptions we have computed the solution of the equations and run simulations for the same progenitor and engine parameters.

\footnotetext{
${ }^{2}$ The factor of two is calibrated through simulations. Note that this approximation is different from that of LB05, who assumed a constant aperture of the cocoon.
} 
Figure 18 shows a comparison of the simulation results (model 10g5) with the semi-analytic predictions. During the confined jet phase, before the breakout, the motion of the head of the jet is reproduced with reasonable accuracy. The energy stored in the cocoon at the time of breakout and the energy given to the stellar material (potentially powering a supernova explosion) are accurate within $10 \%$, while the breakout time is reproduced within $20 \%$. Comparison with other simulations (16TIg5, t10g2 and t5g2) show that the semi-analytic results are accurate to within $20 \%$ with respect to the numerical treatment. The analytic treatment tends to be more accurate for jets injected with higher values of $\gamma \theta_{0}$, i.e., jets that have lost causal contact. This is not surprising since our analytic treatment does not account for the spreading of the jet due to internal motions, which is relevant in the $\gamma<\theta_{0}^{-1}$ case.

\section{Afterglows}

Besides light curves of the prompt emission (Fig. 12), we can compute afterglow light curves based on the energy distributions obtained from these simulations. To this aim, we adopt the afterglow code of Rossi et al. (2004). We input into the code the energies from model 16TIg5 and t $10 \mathrm{~g} 5$ with a lower-limit on the Lorentz factor $\gamma_{\infty}=5$ and we assume a constant density of the ISM with $n=10 \mathrm{~cm}^{-3}$. The flow is supposed to propagate from the low radii of our outer boundary to the external shock radius without re-adjusting the energy distribution and no sideways expansion of the external shock is assumed. We also simplify the computation by assuming a Lorentz factor $\gamma=400$ for the whole fireball. This simplification is due to the fact that it is not possible to input, for a given angle, material with different Lorentz factors, as obtained in the simulations. The effect of this approximation should be to slightly modify the shape of the light curve around the peak of the afterglow emission.

Results of the afterglow calculation are shown in Fig. 19, Light curves have been computed for different observing off-axis angles $\theta_{0}=0,2,4,8,16$, and 32 degrees and for two simulations: model 16TIg5 and model t10g5. The jet properties are the same in the two models, but the progenitor stars are different. In each panel we plot also the afterglow from a standard top-hat jet for comparison. The behavior of the two simulations is quite different. Model 16TI has a very flat energy distribution in its center. For this reason, the inner light curves resemble very well those of a top hat jet. Only the on-axis light curve deviates due to its high energy. While the slopes are different from those of the top-hat afterglow, the spectra are the same. This implies that the use of the so-called afterglow closure relations 3 (Price et al. 2002) cannot be blindly applied to afterglow with the angular energy distributions derived from these simulations.

Owing to its more centrally condensed distribution, model t10g5 has afterglows for small off-

\footnotetext{
${ }^{3}$ Closure relations are simple equations that associate a temporal decay of the afterglow to a given spectral slope and to a given distribution of the ambient medium into which the external shock runs.
} 
axis angles that differ from each other and from the top hat example. Qualitatively, the curves are however similar to one another, with an early shallow decay followed by a sharper decay when the jet reaches causal contact. In both models $16 \mathrm{TI}$ and t10g5, curves at large off-axis angles show a plateau or even a bump several hours to weeks after the explosion. This is due to the fact that the radiation from the brighter jet core enters into the line of sight at late times. Comparison of the two lower panels of Fig. 19 teaches us how the progenitor structure is important not only for the prompt GRB emission, but also for the ensuing afterglow radiation.

\section{Summary and Conclusions}

We have presented high resolution 2D simulations of the propagation of light relativistic jets inside the cores of massive stars. We use an adaptive mesh code (FLASH) that allows us to study the behavior of the jet-star interaction over a long timescale and a wide spatial range. Different stellar progenitors as well as initial conditions of the jet are explored. Thanks to the high resolution and large spatial and temporal domain of our simulations we can confirm and study in more detail jet features discussed in previous work (MacFadyen \& Woosley 1999; Aloy 2000; MacFadyen et al. 2001; Zhang et al. 2003, 2004; Mizuta et al. 2006), as well as identify new phenomenologies.

The main conclusion of this paper is that, even if the central engine is stationary, the jet that propagates out of the progenitor star is characterized by three phases, all of which display significant variability. The first phase is a wide angle release of mildly to moderately relativistic material, which we call the precursor. This phase is due to the release of the turbulent shocked material that accumulates around the jet due to vortex shedding before it breaks out of the star (Ramirez-Ruiz et al. 2002; Zhang et al. 2003). This initial phase is followed by a shocked jet phase. In this second phase the jet material that flows out of the star has been heavily shocked. The energy flow is highly variable in this phase, and no temporal trend in the properties can be identified. During this phase the jet is most highly collimated. Finally, as the pressure of the cocoon decreases, the jet settles into a stable configuration with a freely expanding core surrounded by a shocked shear layer at the boundary with the cocoon material. In this phase the energy flow is almost constant, and the jet opening angle increases logarithmically with time.

This temporal evolution of the jet is also associated with the angular distribution of energy, and therefore determines what different observers see from different directions. The precursor is characterized by a wide opening angle and can be seen from most directions. The shocked jet phase is the most concentrated and can be seen only by observers within several degrees of the axis. As a consequence, such observers will see a very bright event. Observers who lie a few degrees outside the shocked jet cone will see the precursor, followed by a dead time of several tens of seconds. Eventually, when the jet opens to contain their line of sight, they will see a second phase of emission. These observers will therefore measure dead-times much longer than any timescale of the inner engine, as found in several BATSE light curves (Lazzati 2005). 
The overall angular distribution of energy is complex and does not seem to follow any simple correlation with the jet or progenitor properties. In some cases, the jet is characterized by a flat core with a sudden cutoff, very similar to a top-hat jet (16TIg5), while in other cases the distribution is more centrally peaked. It seems, however, that our high resolution simulations were not able to reproduce previous analytic or numerical results like the $\theta^{-2}$ universal jet of LB05 or the $\theta^{-3}$ distribution found by Zhang et al. (2003). Such behaviors are observed only in a limited range of angles from the jet axis. We computed afterglow light curves from our energy distributions showing that differences in the afterglow phase can result from the different properties of the progenitor and/or of the jet in the core of the star. We also developed further the analytic model of LB05, refining some approximations to obtain a model that can reproduce such basic features of the simulations as the propagation of the bow shock inside the star and the precursor energy, as well as the qualitative evolution of the jet opening angle.

It is easy to find paths along which this work can be developed further. Higher resolution and dimensionality will certainly be worth exploring (Zhang et al. 2004), as well as the consequences of a more accurate equation of state capable of describing both relativistic and non-relativistic material. Including a non-relativistic wind from the accretion disk is also worthwhile, as this seems to be a ubiquitous outcome of jet-launching simulations (Proga et al. 2003; de Villiers et al. 2006). This wind component may alter the cocoon properties, which are so important in defining the behavior of the precursor and shocked jet phases. Simulations also suggest that the jet luminosity should be highly variable if not intermittent. Preliminary studies (Aloy et al. 2000) suggest that this may enhance the propagation of the jet through the star, but higher resolution simulations are required. An interesting hypothesis is that the three phases of jet propagation will respond differently to an intermittent engine, with the precursor virtually unaffected while the unshocked jet should retain most of the engine variability.

The software used in this work was in part developed by the DOE-supported ASC/Alliance Center for Astrophysical Thermonuclear Flashes at the University of Chicago. We thank Alex Heger for providing us with the tabulated properties of his stellar models, Andrew MacFadyen for useful discussions and advice on the testing of the relativistic FLASH code and Miguel Aloy for

useful discussions. This work was supported by NSF grant AST-0307502, NASA Astrophysical Theory Grant NNG06GI06G, and Swift Guest Investigator Program NNX06AB69G.

\section{REFERENCES}

Aloy, M. A., Müller, E., Ibáñez, J. M., Martí, J. M., \& MacFadyen, A. 2000, ApJ, 531, L119

Begelman, M. C., \& Cioffi, D. F. 1989, ApJ, 345, L21

Del Zanna, L., \& Bucciantini, N., 2002, A\&A, 390, 1177

De Villiers, J.-P., Staff, J. \& Ouyed, R., 2006, ApJ submitted (astro-ph/0502225) 
Emery, A. E., 1968, J. Comput. Physics, 2, 306

Galama, T. J., et al., 1998, Nature, 395, 670

Heger, A., Woosley, S. E., \& Sqruit, H. C., 2005, ApJ, 626, 350

Lazzati, D., 2005, MNRAS, 357, 2, 722

Lazzati, D. \& Begelman, M. C., 2005, ApJ, 629, 903 (LB05)

Lazzati, D., Begelman, M. C., Ghirlanda, G., Ghisellini, G., \& Firmani, C., 2005, Proceedings of the 22nd Texas Symposium on Relativistic Astrophysics and Cosmology, astro-ph/0503630

Lithwick, Y., \& Sari, R. 2001, ApJ, 555, 540

Lucas-Serrano, A., Font, J. A., Ibáñez, J. M., Martí, J. M., 2004, A\&A, 428, 703

MacFadyen, A., \& Woosley, S. E., 1999, ApJ, 524, 262

MacFadyen, A., Woosley, S. E. \& Heger, A., 2001, ApJ, 550, 410

Martí, J. M., Müller, E., Font, J. A., Ibáñez, J. M., and Marquina, A., 1997, ApJ, 479, 151

Matzner, C. D. 2003, MNRAS, 345, 575

Mignone, A., Plewa, T., \& Bodo, G., 2005, ApJS, 160, 199

Mizuno, Y., Yamada, S., Koide, S., \& Shibata, K. 2004, ApJ, 615, 389

Mizuta, A., Yamada, S., \& Takabe, H. 2004, ApJ, 606, 804

Mizuta, A., Yamasaki, T., Nagataki, S., \& Mineshige, S. 2006, ApJ in press (astro-ph/0607544)

Nagataki, S., Takahashi, R., Mizuta, A. \& Takiwaki, T., 2006, ApJ submitted (astro-ph/0608233)

Price, P. A., et al. 2002, ApJ, 572, L51

Proga, D., MacFadyen, A. I., Armitage, P. J., \& Begelman, M. C. 2003, ApJ, 599, L5

Pons, J. A., \& Martí, J., \& Müller, E., 2000, Journal of Fluid Mechanics, 422, 1, 125

Proga, D., MacFadyen, A. I., Armitage, P. J., \& Begelman, M. C., 2003, ApJ, 599, L5

Ramirez-Ruiz, E., Celotti, A., \& Rees, M. J., 2002, MNRAS, 337, 4, 1349

Rossi, E., Lazzati, D., \& Rees, M. J., 2002, MNRAS, 337, 4, 1349

Rossi, E., Lazzati, D., Salmonson, J. D., \& Ghisellini, G., 2004, MNRAS, 354, 86

Scheck, L., Aloy, M. A., Martí, J. M., Gómez, J. L., Müller, E. 2002, MNRAS, 331, 615 
Waxman, E. 2004, ApJ, 602, 886

Woodward, P. \& Colella, P., 1984, J. Comput. Physics, 23, 276

Woosley, S. E. \& Heger, A., 2006, ApJ, 637, 914

Zhang, W., \& MacFadyen, A. I. 2006, ApJS, 164, 255 (RAM)

Zhang, W., Woosley, S. E., \& MacFadyen, A. I., 2003, ApJ, 586, 356

Zhang, W., Woosley, S. E., \& Heger, A. I., 2004, ApJ, 608, 365 
Table 1. Model Parameters

\begin{tabular}{lllll}
\hline \hline Model & Stellar Model & Input Power $\left(\mathrm{erg} \mathrm{s}^{-1}\right)$ & Injection Opening Angle & Injection Lorentz \\
\hline 16TIg5 & Realistic & $5.32 \times 10^{50}$ & $10^{\circ}$ & 5 \\
16TIg2 & Realistic & $5.32 \times 10^{50}$ & $10^{\circ}$ & 2 \\
t10g5 & Power Law & $5.32 \times 10^{50}$ & $10^{\circ}$ & 5 \\
t10g2 & Power Law & $5.32 \times 10^{50}$ & $10^{\circ}$ & 2 \\
t5g2 & Power Law & $5.32 \times 10^{50}$ & $5^{\circ}$ & 2 \\
t20g5 & Power Law & $5.32 \times 10^{50}$ & $20^{\circ}$ & 5 \\
\hline
\end{tabular}

Table 2. Breakout Times of Cocoon, Shocked Jet and Unshocked Jet at Surface of Star

\begin{tabular}{llll}
\hline \hline \multicolumn{1}{c}{ Model } & Cocoon Breakout $(\mathrm{s})$ & Shocked Jet Breakout $(\mathrm{s})$ & Unshocked Jet Breakout (s) \\
\hline 16TIg5 & 7.53 & 7.73 & 23.4 \\
16TIg2 & 9.66 & 12.0 & 25.0 \\
t10g5 & 13.1 & 14.0 & 29.0 \\
t10g2 & 24.0 & 31.0 & 48.0 \\
t5g2 & 21.2 & 31.8 & 46.0 \\
16TIg5lowres & 4.93 & 5.00 & 23.9 \\
\hline
\end{tabular}

Table 3. Precursor Energetics. Columns report the model identification, the energy emitted with $\gamma_{\infty}>10$, the angle at which the energy is maximized and the width of $d E / d \Omega$ at one tenth of its maximum, the energy emitted with $\gamma_{\infty}>50$, the angle at which the energy is maximized and the width of $d E / d \Omega$ at one tenth of its maximum. The last column reports the ratio of the energies for the two limiting Lorentz factors.

\begin{tabular}{llllllll}
\hline \hline Model & $E_{\gamma_{\infty} \geq 10}(\mathrm{erg})$ & $\theta_{\max }$ & $\theta_{\frac{1}{10}}$ & $E_{\gamma_{\infty} \geq 50}(\mathrm{erg})$ & $\theta_{\max }$ & $\theta_{\frac{1}{10}}$ & $E_{\gamma_{\infty} \geq 50} / E_{\gamma_{\infty} \geq 10}$ \\
\hline 16TIg5 & $3.467 \times 10^{50}$ & $4^{\circ}$ & $10^{\circ}$ & $2.470 \times 10^{50}$ & $4^{\circ}$ & $9^{\circ}$ & 0.712 \\
16TIg2 & $4.563 \times 10^{51}$ & $15^{\circ}$ & $34^{\circ}$ & $2.613 \times 10^{51}$ & $15^{\circ}$ & $14^{\circ}$ & 0.573 \\
t10g5 & $7.948 \times 10^{50}$ & $15^{\circ}$ & $54^{\circ}$ & $4.602 \times 10^{50}$ & $15^{\circ}$ & $14^{\circ}$ & 0.579 \\
t10g2 & $1.181 \times 10^{51}$ & $23^{\circ}$ & $34^{\circ}$ & $5.281 \times 10^{50}$ & $23^{\circ}$ & $24^{\circ}$ & 0.447 \\
t5g2 & $2.560 \times 10^{51}$ & $7^{\circ}$ & $39^{\circ}$ & $1.898 \times 10^{51}$ & $7^{\circ}$ & $39^{\circ}$ & 0.741 \\
\hline
\end{tabular}




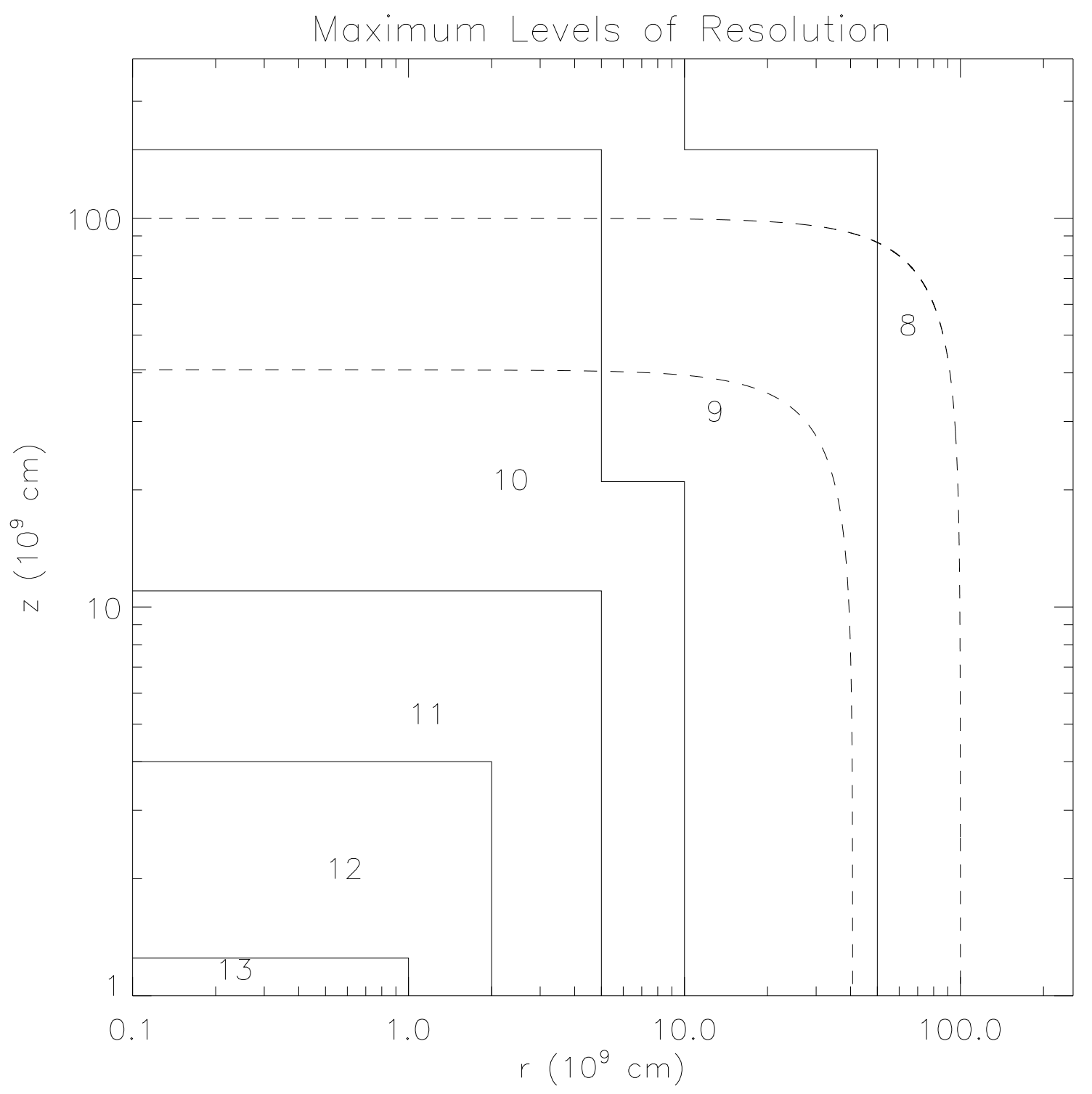

Fig. 1.- Maximum level of allowed resolution in different grid regions. Each level down represents a factor of 2 decrease in maximum resolution. Level 8 resolution corresponds to a pixel size of $2.5 \times 10^{8} \mathrm{~cm}$. The two dotted lines are at a radius of $10^{11} \mathrm{~cm}$ and $4.077 \times 10^{10} \mathrm{~cm}$, the radii of the two stellar models used. Note that the axes, as plotted, are logarithmic in order to emphasize the regions close to the center of the star, and that the horizontal axis does extend down to 0, rather than $10^{8} \mathrm{~cm}$ as plotted, while the vertical axis begins at $10^{9} \mathrm{~cm}$ above the equator of the star. 

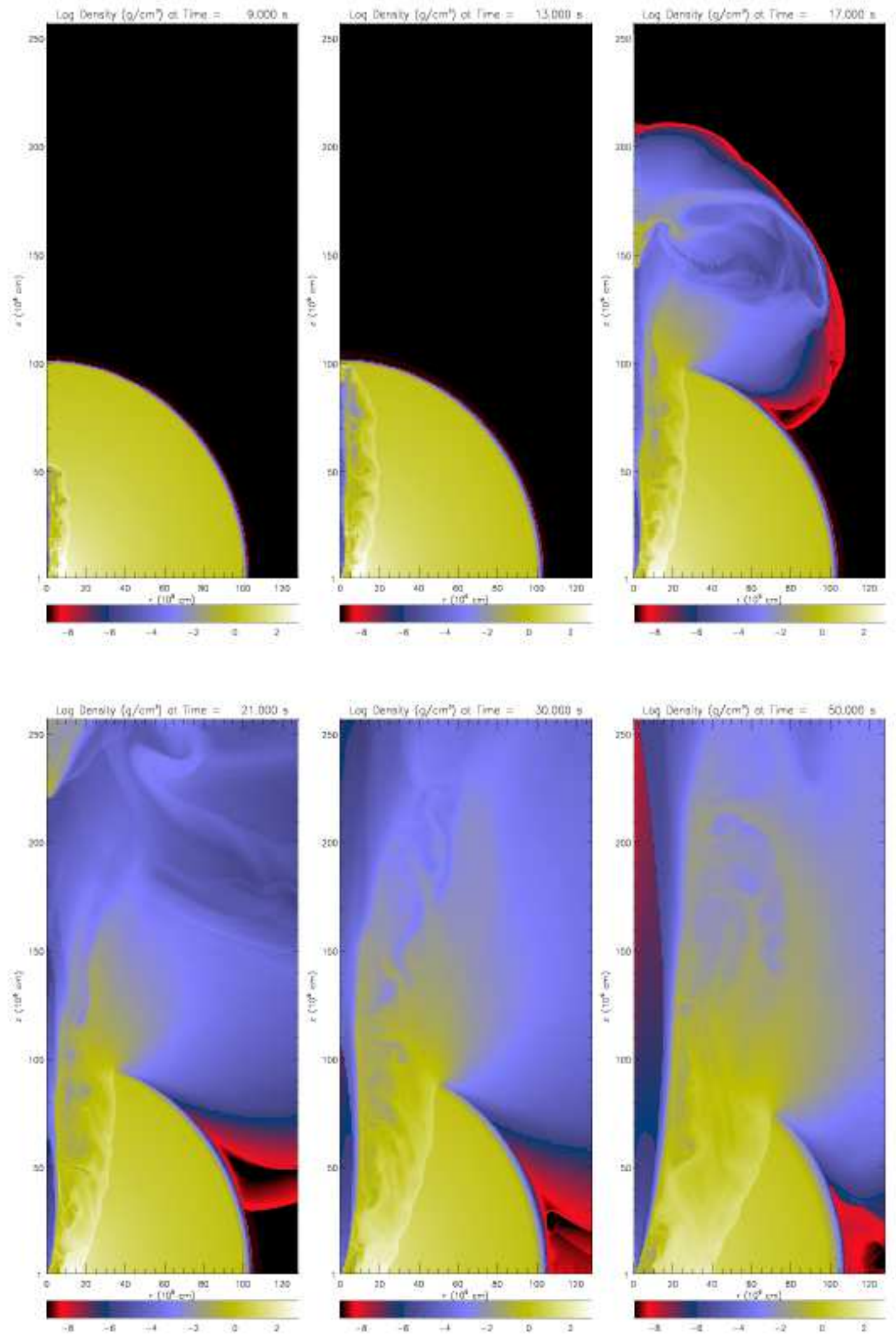

Fig. 2.- Time sequence of logarithmic density for model t10g5 from 9 to 50 seconds. Initially the jet is confined, and hot turbulent material is stored in a cocoon (first two panels). When the jet head reaches the surface, the cocoon is released as a wide angle outflow (third panel). Immediately afterwards, a heavily shocked jet flows outside the star (third and fourth panels). Eventually, a more stable configuration emerges (fifth and sixth panels) in which the jet is internally free-flowing, and is bounded by a shear layer at the contact discontinuity with the star. 

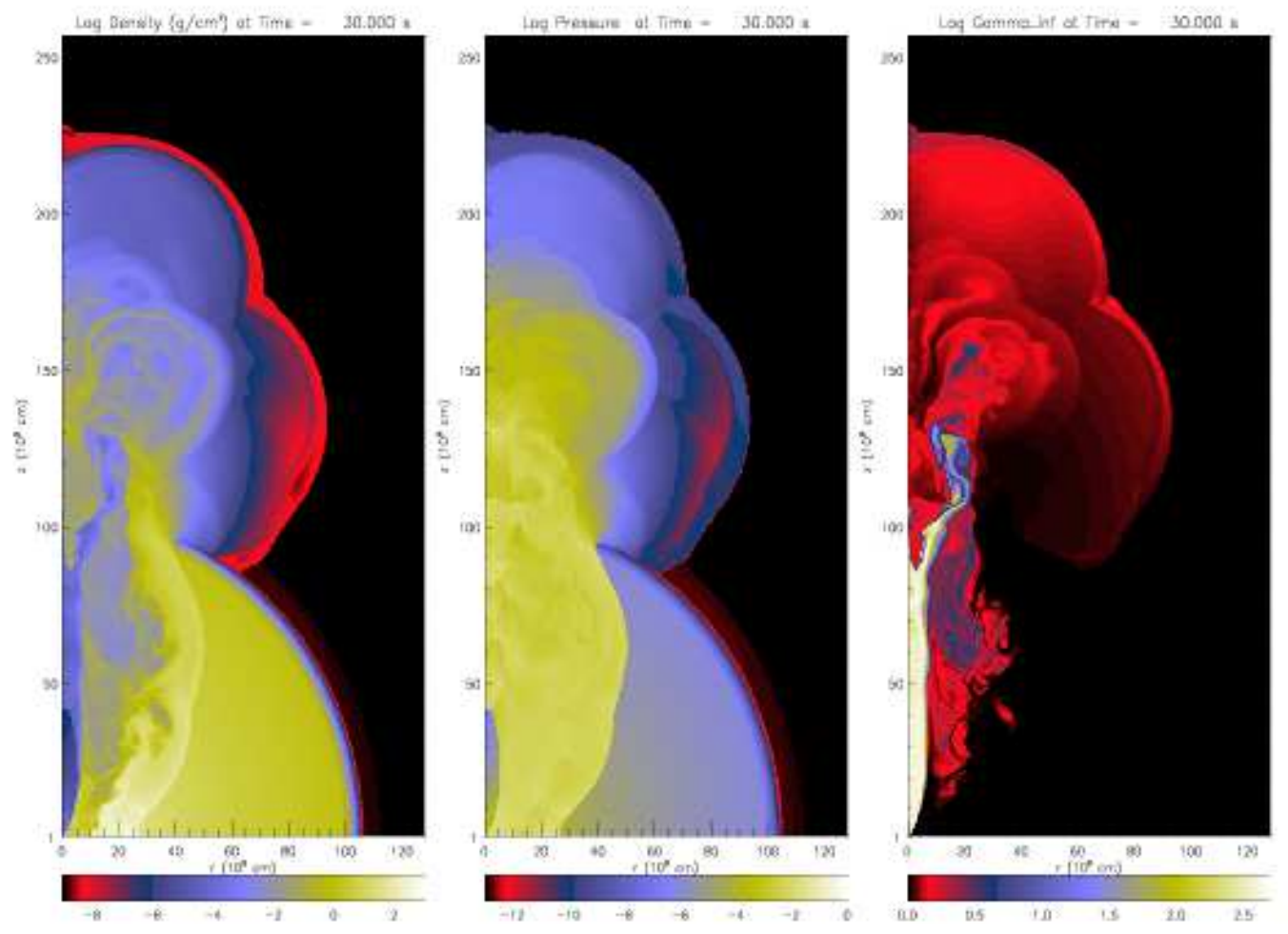

Fig. 3. - Logarithmic density, pressure and $\gamma_{\infty}$ (left to right) for model t10g2 at 30 seconds. Visible are the cocoon (high density, high pressure, low $\gamma_{\infty}$ ), precursor region (high $\gamma_{\infty}$, off axis), shocked jet (low density, high pressure, high $\gamma_{\infty}$ ), and unshocked jet (low density, low pressure, high $\gamma_{\infty}$ ). 


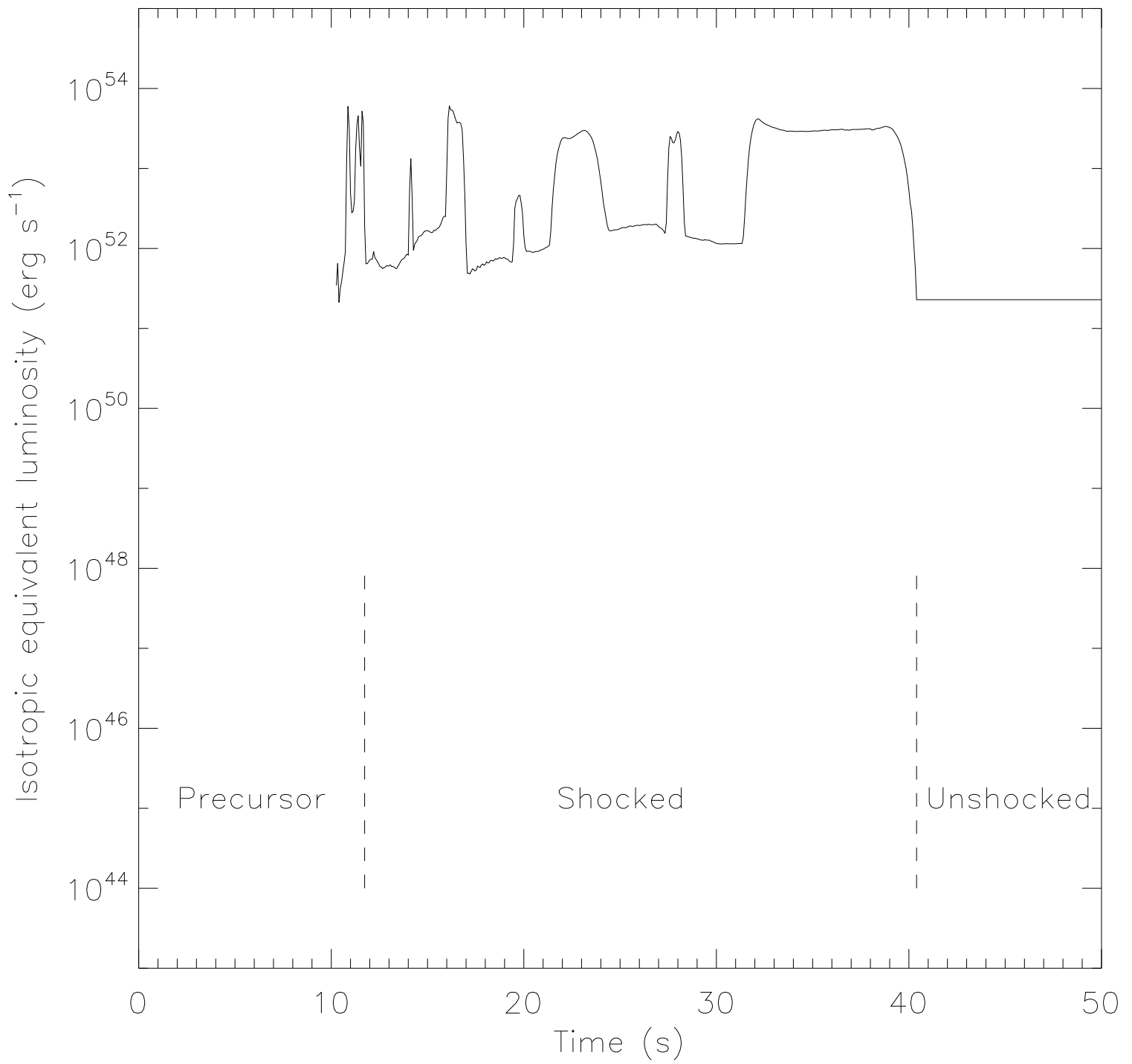

Fig. 4. - The definition of the transition times between the three phases of jet evolution described in the text. The figure shows the energy flux along the jet axis versus time for model 16TIg5. The different phases of the jet are shown. 

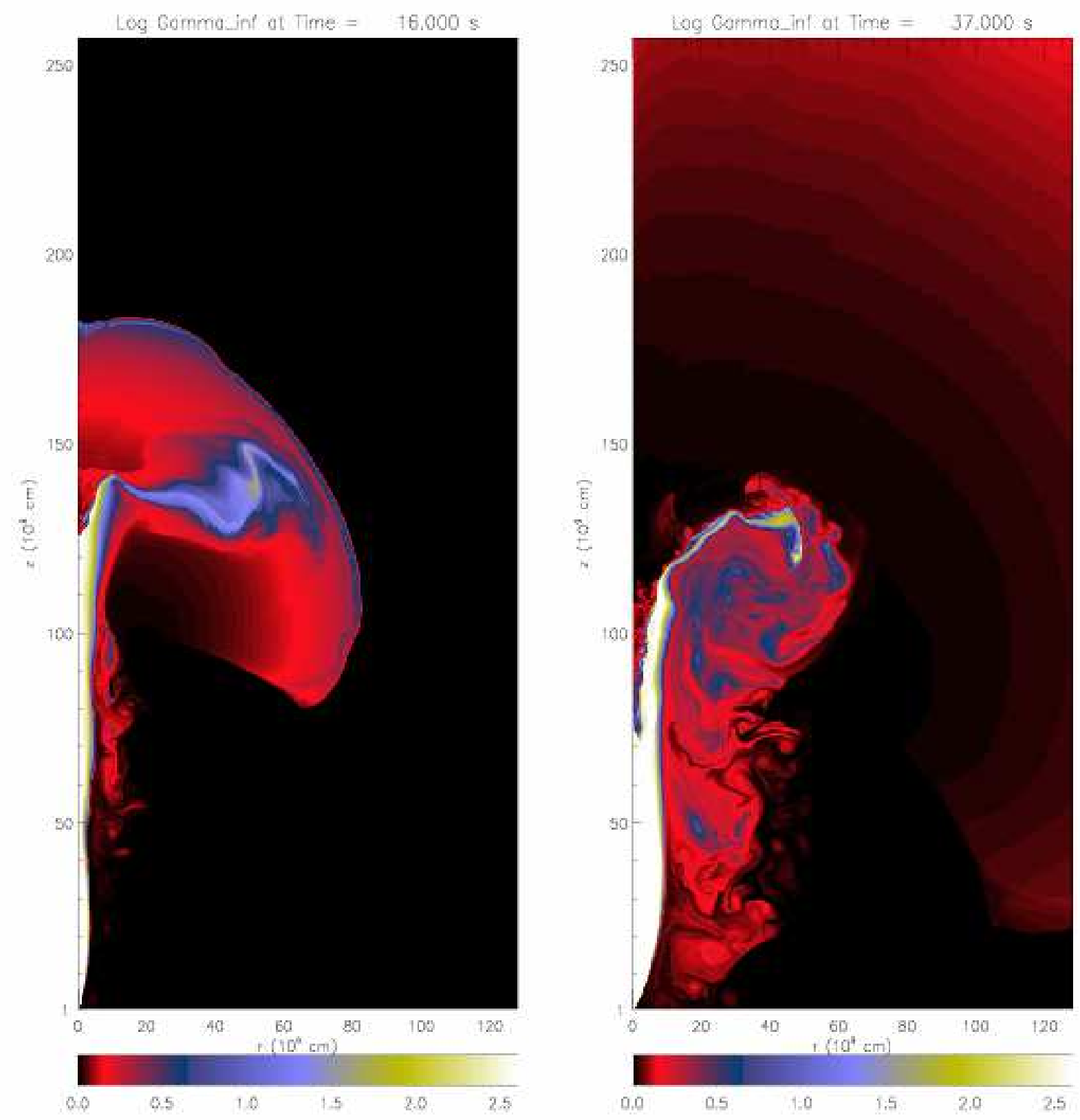

Fig. 5.- Comparison of $\gamma_{\infty}$ for models t10g5 at 16 seconds (left) and t20g5 at 37 seconds (right). Model t20g5 is much less collimated and the relativistic material is being highly deflected even at this late time. 

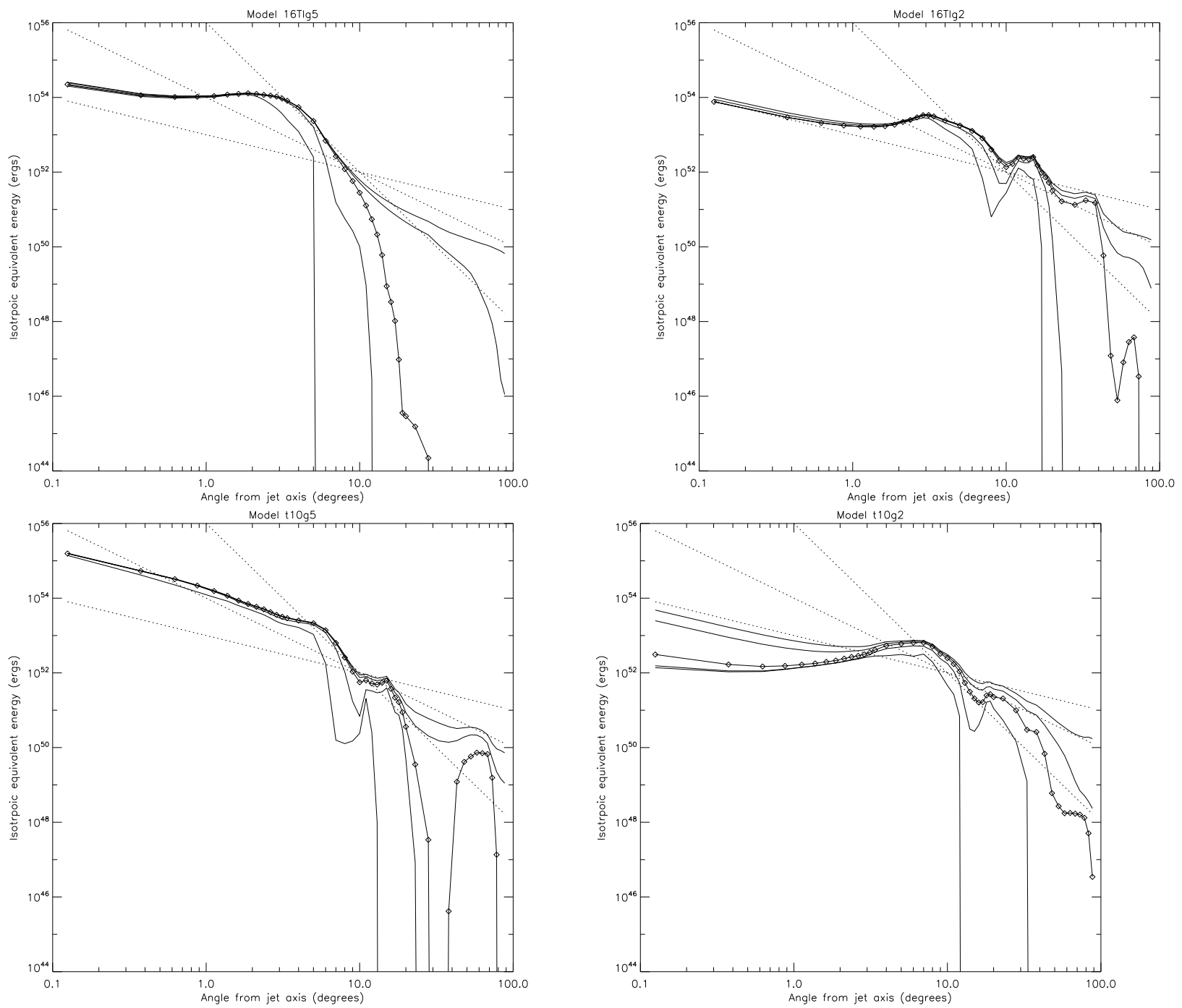

Fig. 6.- Total isotropic equivalent energy as a function of angle for models 16TIg5 (upper left), 16TIg2 (upper right), t10g5 (lower left), and t10g2 (lower right). Different lines correspond to the amount of energy above a minimum Lorentz factor of $\gamma_{\infty}=1.01,2,10,50$, and 200, from top to bottom. Highlighted line corresponds to $\gamma_{\infty}=10$. Dotted lines are slopes of $E_{i s o} \propto \theta^{-1}, \theta^{-2}$, and $\theta^{-4}$. 

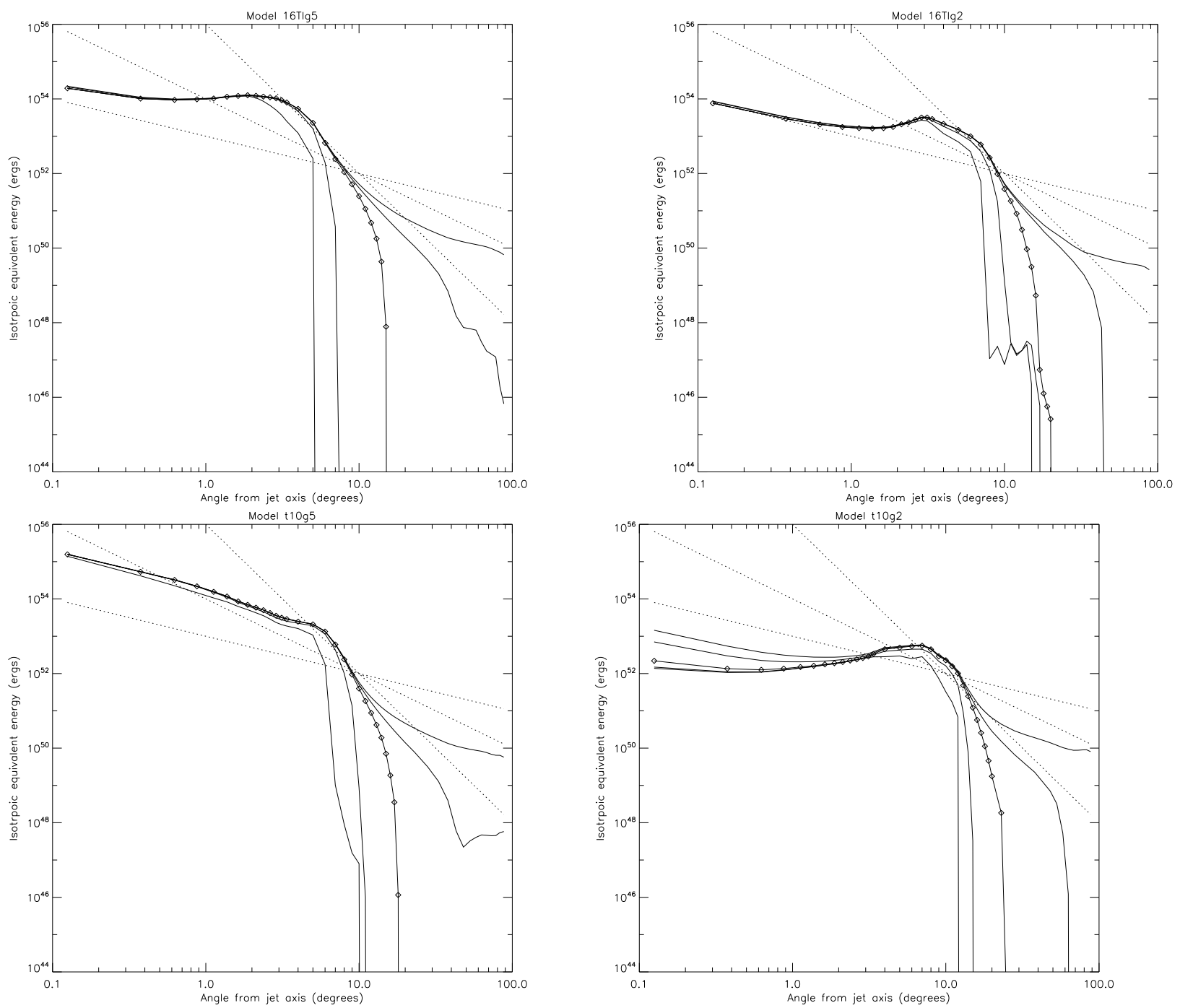

Fig. 7.- Isotropic equivalent energy, excluding precursor phase energy, as a function of angle for models 16TIg5 (upper left), 16TIg2 (upper right), t10g5 (lower left), and t10g2 (lower right). Different lines correspond to the amount of energy above a minimum Lorentz factor of $\gamma_{\infty}=1.01$, $2,10,50$, and 200 , from top to bottom. Highlighted line corresponds to $\gamma_{\infty}=10$. Dotted lines are slopes of $E_{\text {iso }} \propto \theta^{-1}, \theta^{-2}$, and $\theta^{-4}$. 


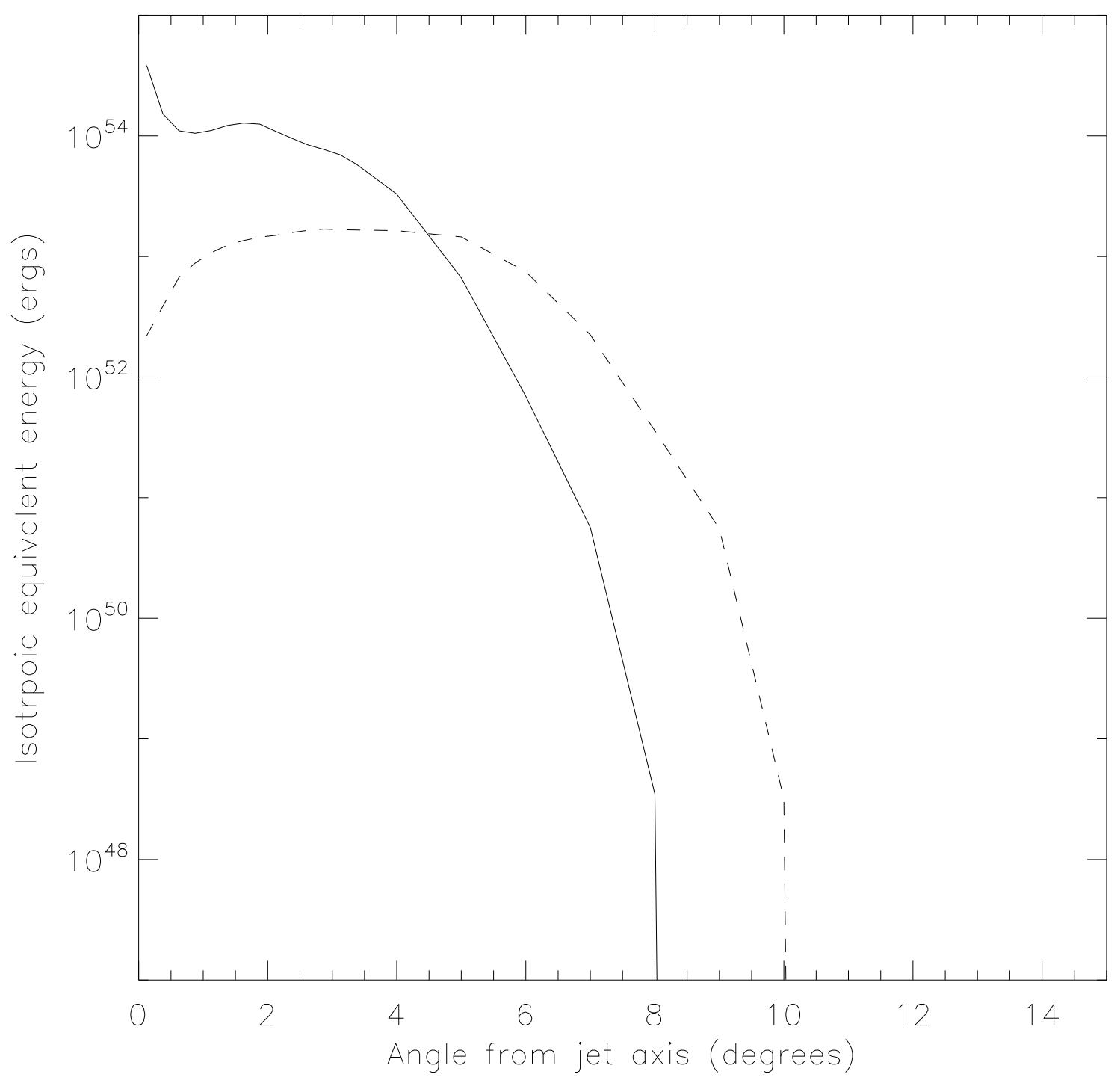

Fig. 8.- Isotropic equivalent energy as a function of angle for model 16TIg5 for the shocked (solid line) and unshocked (dashed line) phases. Both lines have a minimum Lorentz factor of $\gamma_{\infty}=50$. 


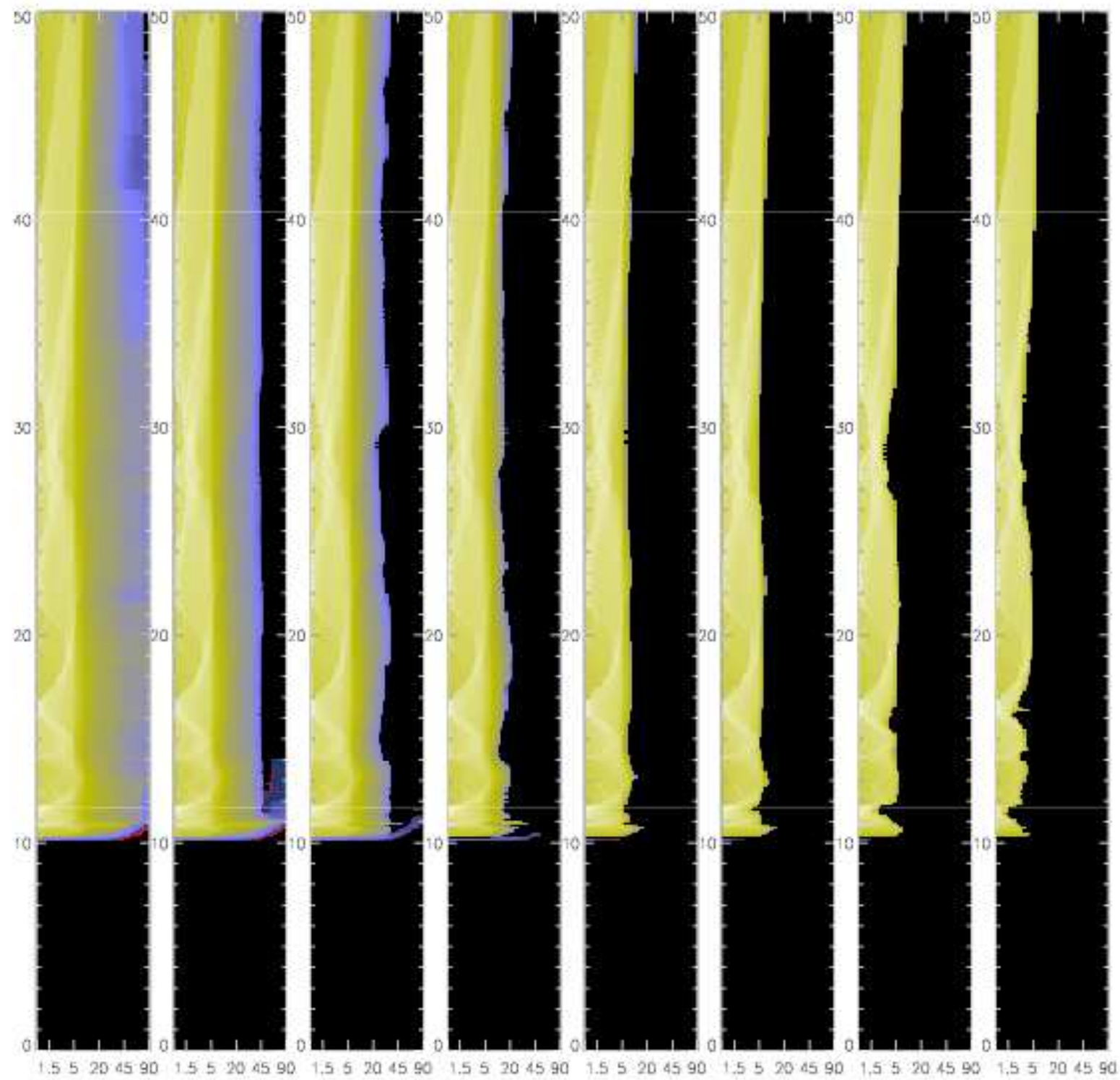

Fig. 9.- Time and angular distribution of (logarithmic) energy from model 16TIg5 measured at $1.2 \times 10^{11} \mathrm{~cm}$. Y-axis is time in seconds and $\mathrm{x}$-axis is angle from jet axis in degrees. The $\mathrm{x}$-axis is not uniform but instead corresponds to the angular bins described in $\S 4.1$. The panels correspond to the amount of energy above a minimum Lorentz factor of $\gamma_{\infty}=1.01,2,5,10,30,50,100$, and 200, from left to right. White horizontal lines show the times at which the transitions between phases take place. 

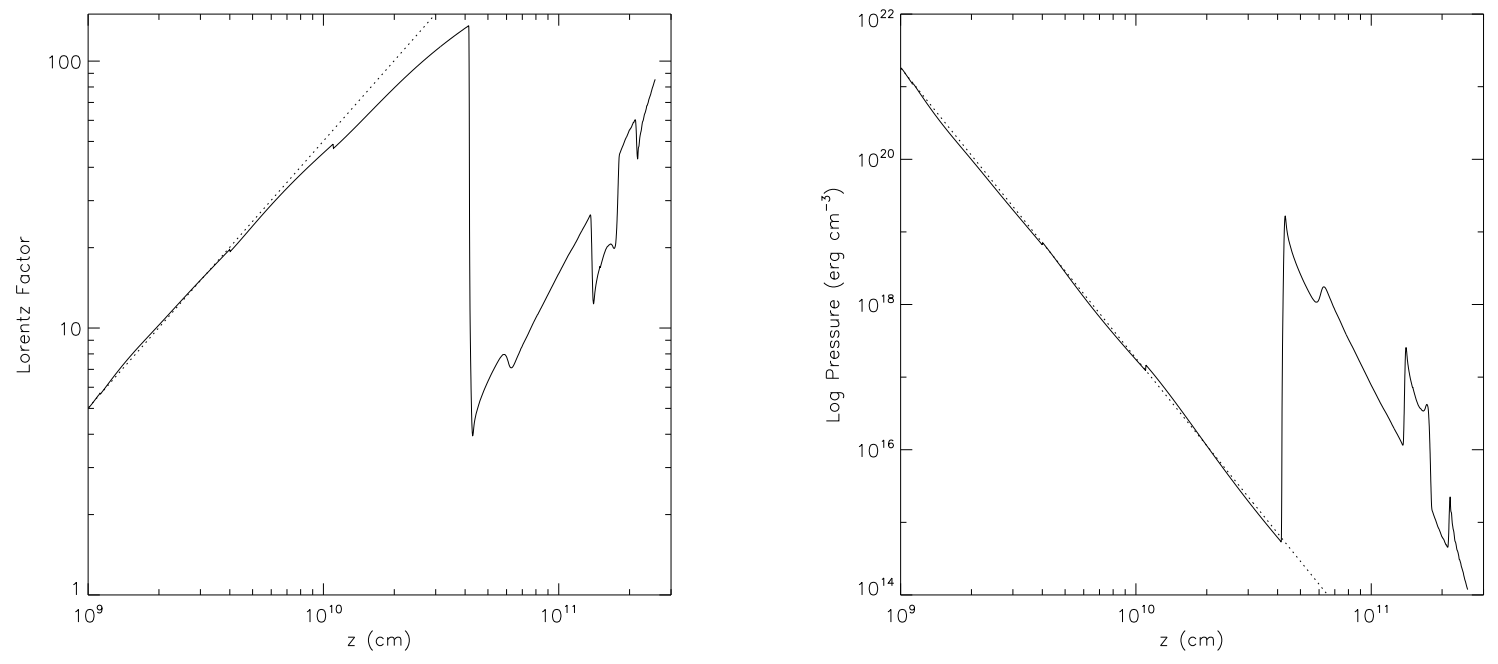

Fig. 10.- Lorentz factor and pressure along the jet axis as a function of the distance from the black hole at the time of the emergence of the unshocked jet phase on the stellar surface for model $16 \operatorname{TIg} 5$. The dotted lines show the theoretical prediction for an free streaming accelerating jet: $\gamma \propto z$ and $p \propto z^{-4}$. This shows that the core of the unshocked jet is free streaming. Small deviations from the theoretical behavior are due to the fact that when $\gamma \gtrsim 100$ the flow is not pressure-dominated any more. 

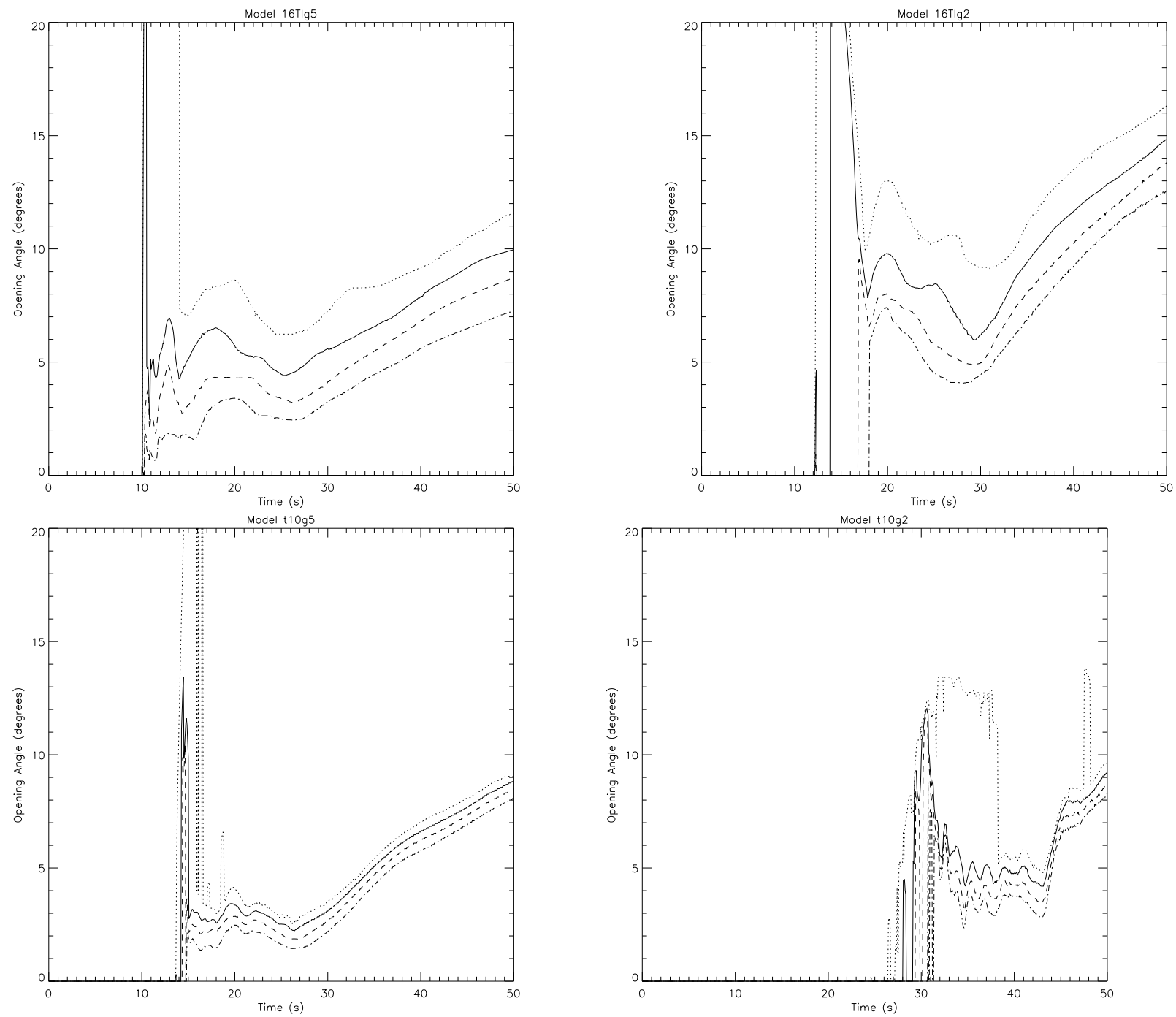

Fig. 11.- Opening angle vs. time at a radius of $1.2 \times 10^{11} \mathrm{~cm}$ for models 16TIg5 (upper left), 16TIg2 (upper right), t10g5 (lower left), and t10g2 (lower right). Different lines correspond to opening angle for material with a minimum terminal Lorentz factor of $\gamma_{\infty}=2$ (dotted line), 10 (solid line), 50 (dashed line), and 200 (dot-dash line). The initially large opening angle is due to the passage of the precursor material. 

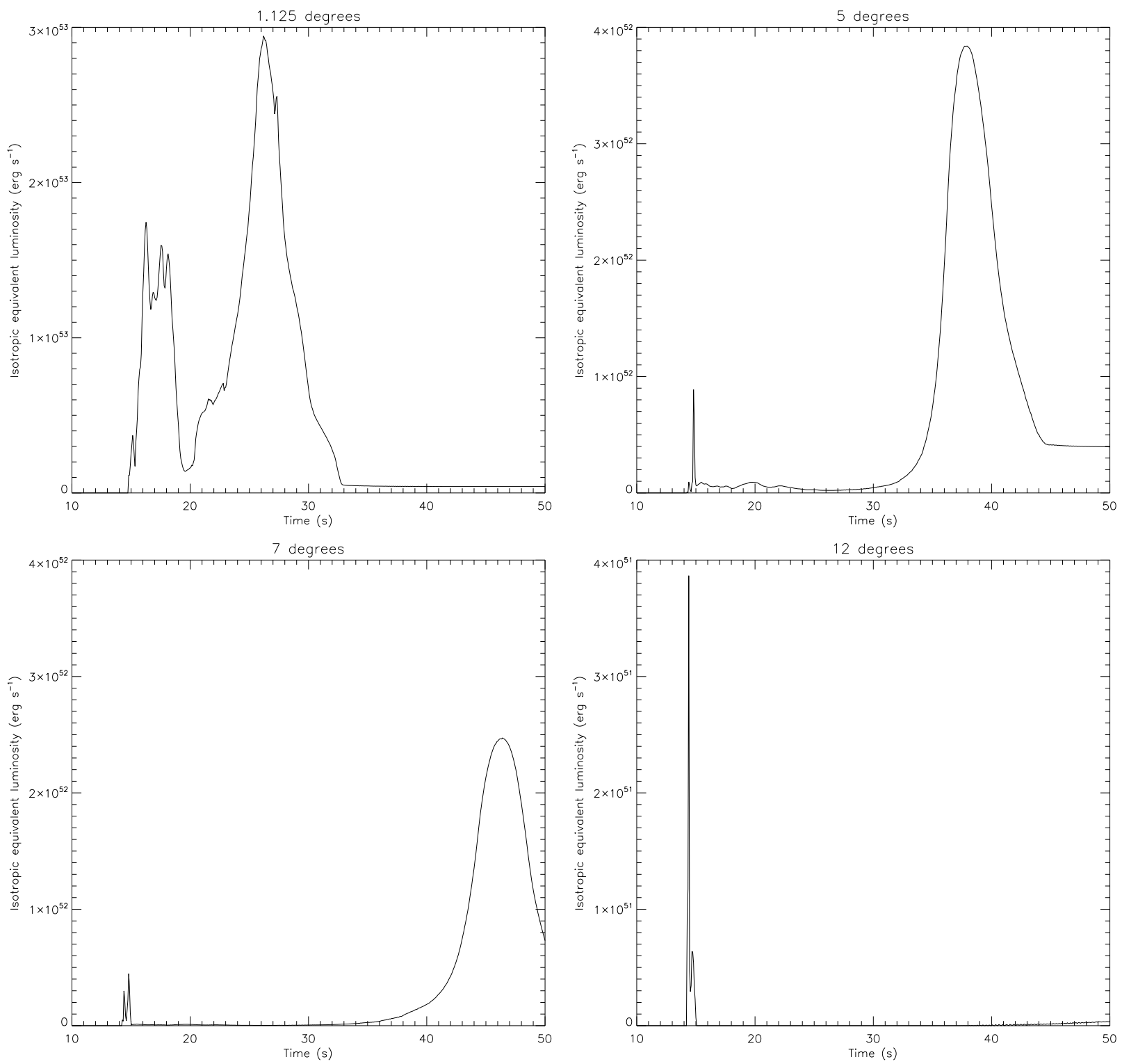

Fig. 12. - Energy flux vs. time for model t $10 \mathrm{~g} 5$ at 4 different angles. The energy flux is for material with a minimum terminal Lorentz factor of $\gamma_{\infty}=10$. Angles represented are $1.125^{\circ}$ (upper left), $5^{\circ}$ (upper right), $7^{\circ}$ (lower left), and $12^{\circ}$ (lower right). 

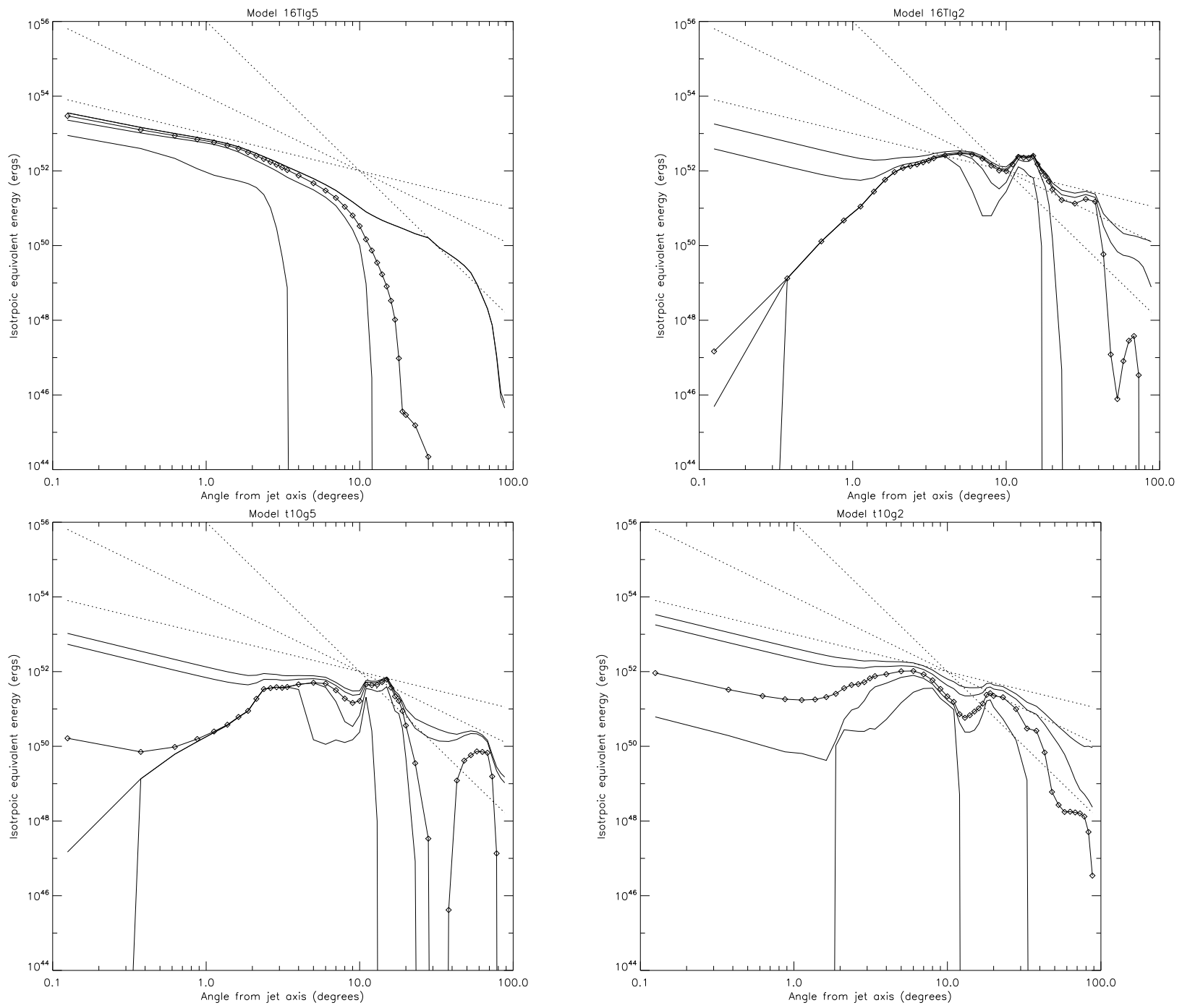

Fig. 13.- Isotropic equivalent energy as a function of angle from precursor phase for models 16TIg5 (upper left), 16TIg2 (upper right), t10g5 (lower left), and t10g2 (lower right). Different lines correspond to the amount of energy above a minimum Lorentz factor of $\gamma_{\infty}=1.01,2,10,50$, and 200, from top to bottom. Highlighted line corresponds to $\gamma_{\infty}=10$. Dotted lines are slopes of $E_{i s o} \propto \theta^{-1}, \theta^{-2}$, and $\theta^{-4}$. 


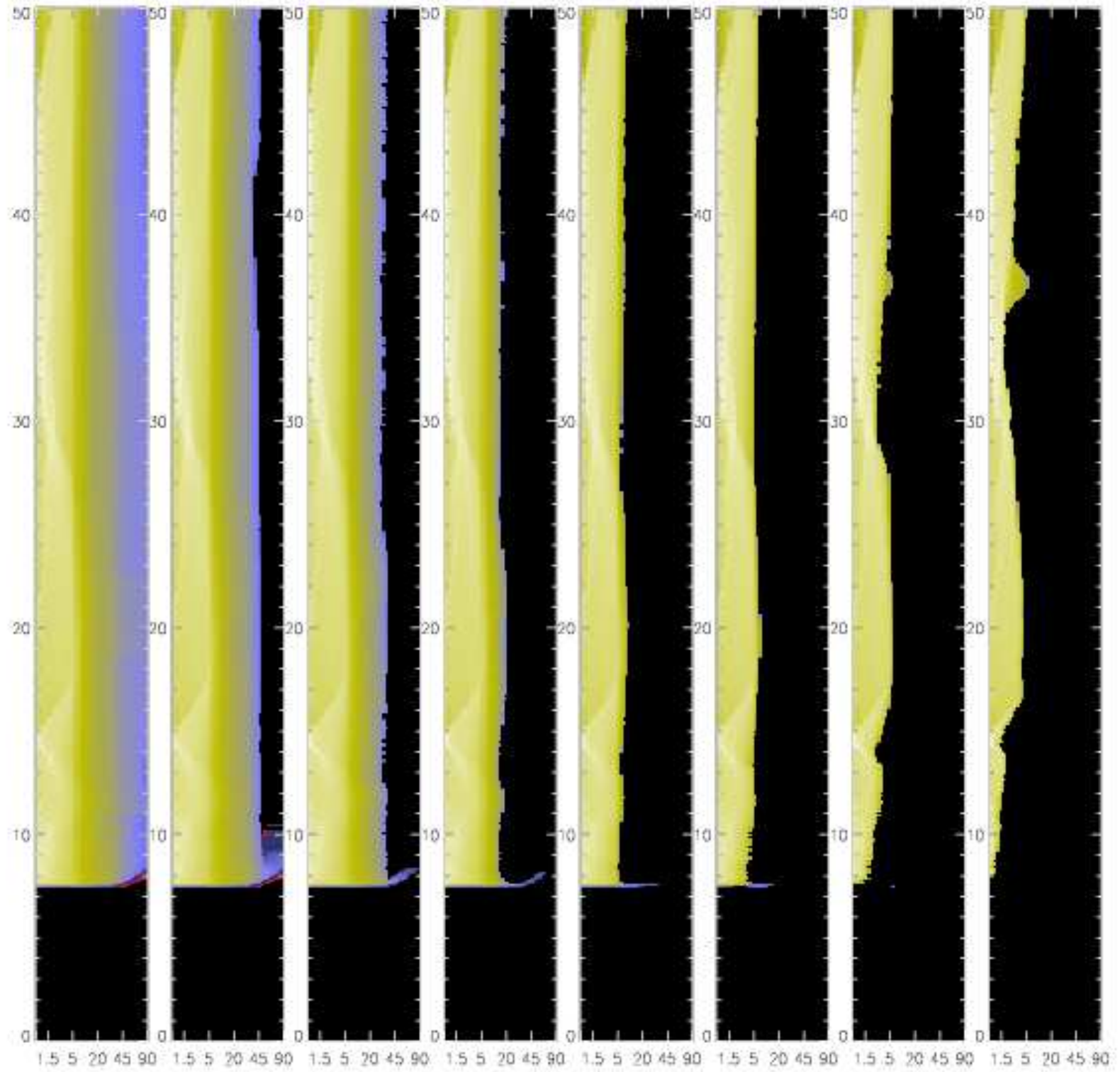

Fig. 14. - Time and angular distribution of (logarithmic) energy from low resolution version of model 16 TIg5 measured at $1.2 \times 10^{11} \mathrm{~cm}$. Y-axis is time in seconds and $\mathrm{x}$-axis is angle from jet axis in degrees. The x-axis is not uniform but instead corresponds to the angular bins described in $\S 4.1$. The panels correspond to the amount of energy above a minimum Lorentz factor of $\gamma_{\infty}=1.01,2$, $5,10,30,50,100$, and 200, from left to right. 

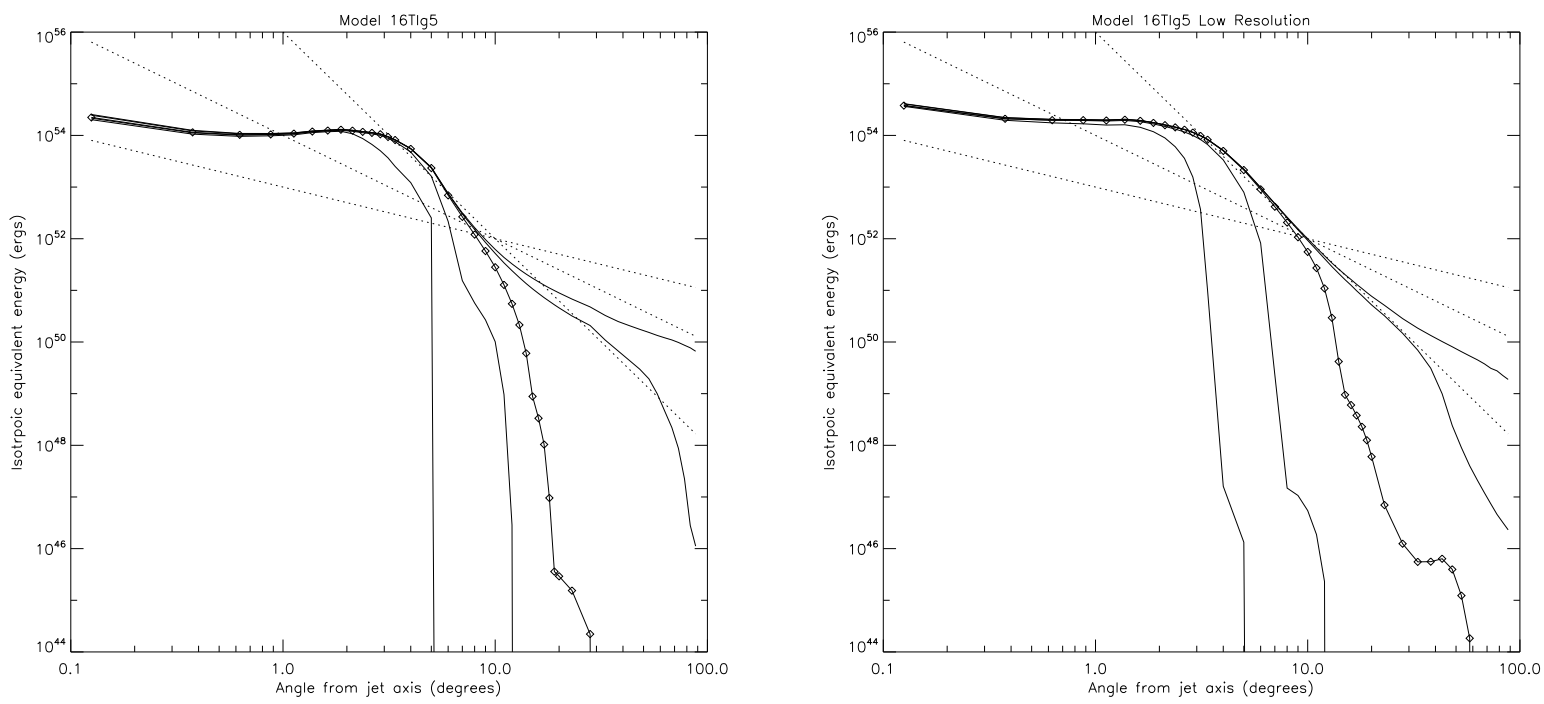

Fig. 15.- Total isotropic equivalent energy as a function of angle for high resolution (left) and low resolution (right) versions of model 16TIg5. Different lines correspond to the amount of energy above a minimum Lorentz factor of $\gamma_{\infty}=1.01,2,10,50$, and 200, from top to bottom. Highlighted line corresponds to $\gamma_{\infty}=10$. Dotted lines are slopes of $E_{i s o} \propto \theta^{-1}, \theta^{-2}$, and $\theta^{-4}$.
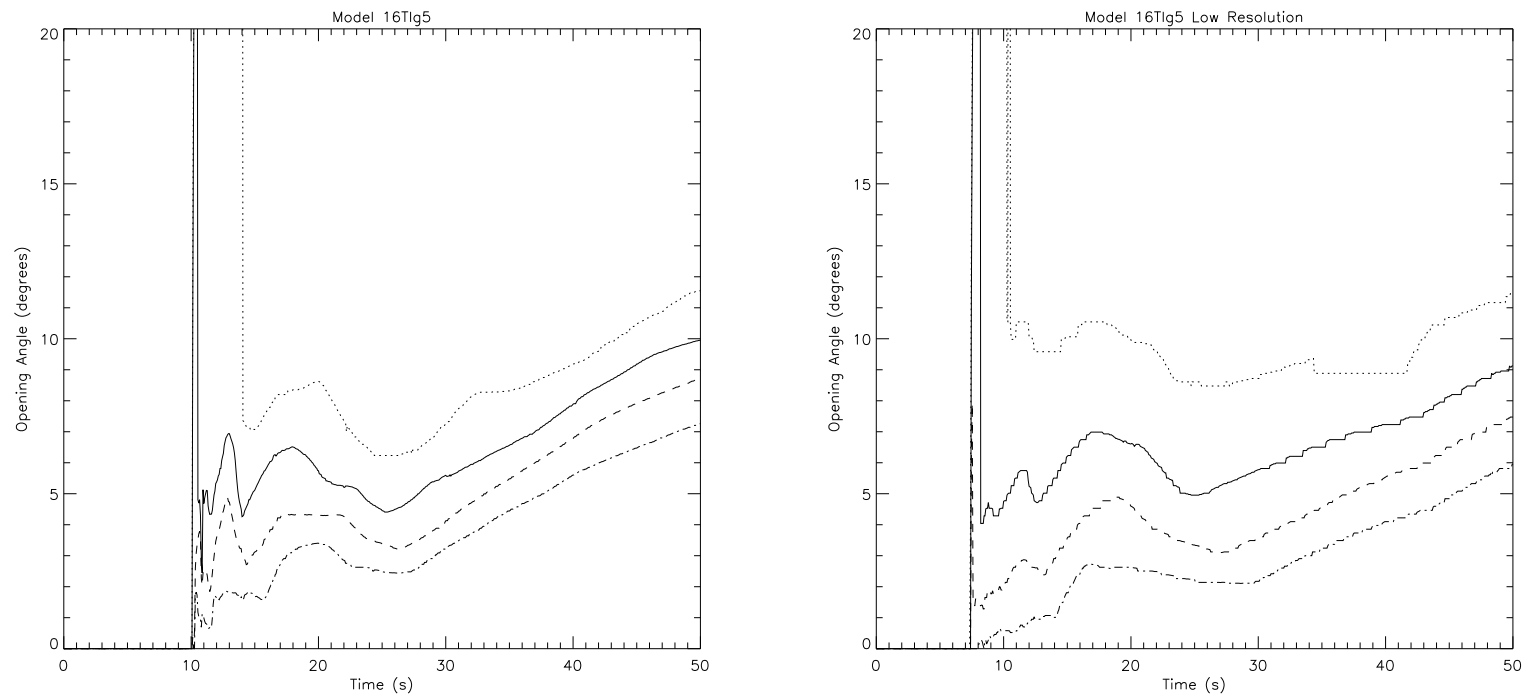

Fig. 16.- Opening angle vs. time for high resolution (left) and low resolution (right) versions of model 16TIg5. Different lines correspond to opening angle for material with a minimum terminal Lorentz factor of $\gamma_{\infty}=2$ (dotted line), 10 (solid line), 50 (dashed line), and 200 (dot-dash line). The initially large opening angle is due to the passage of the precursor material. 


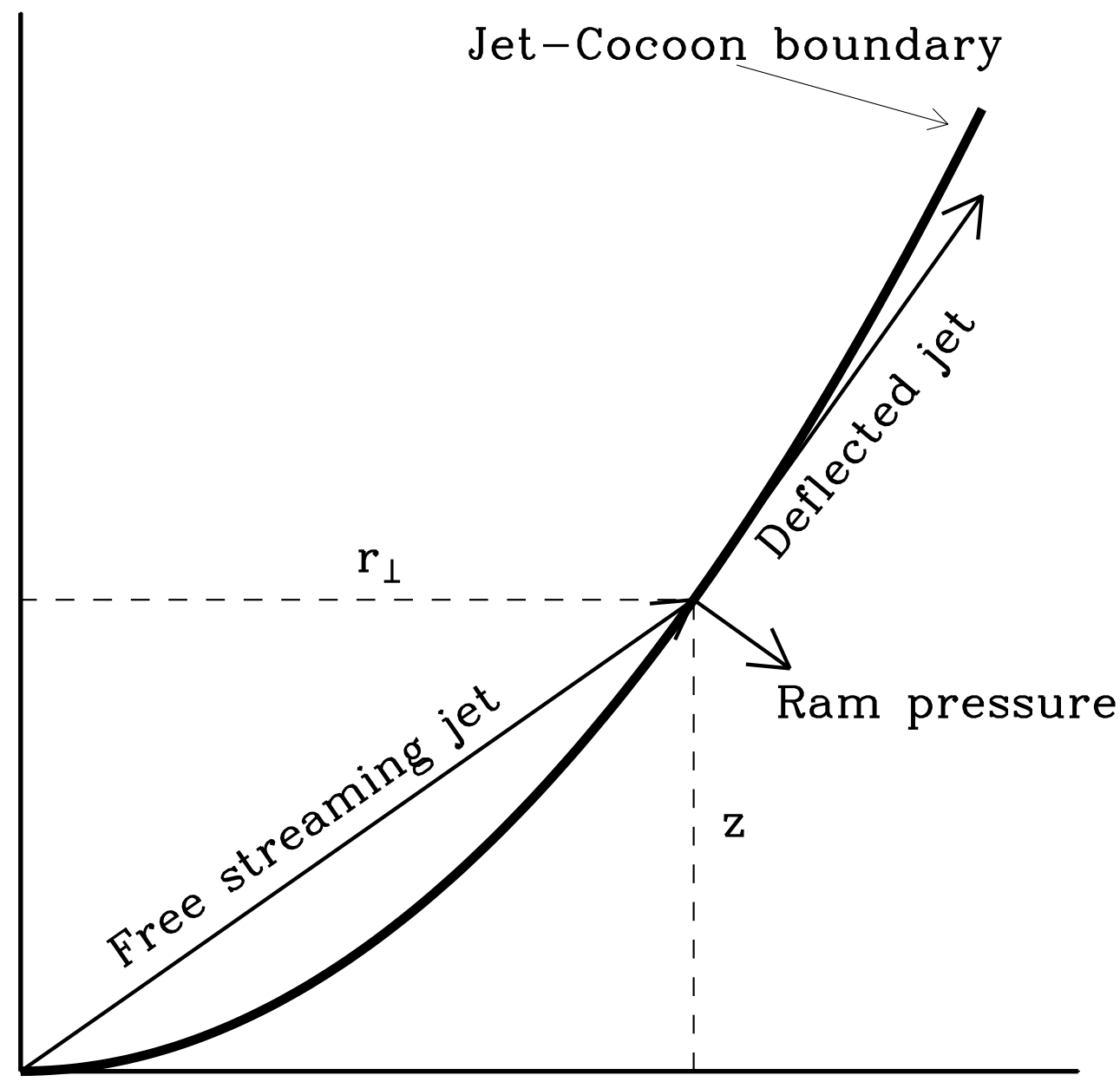

Fig. 17.- Cartoon of the geometry used in the analytic approximations. 


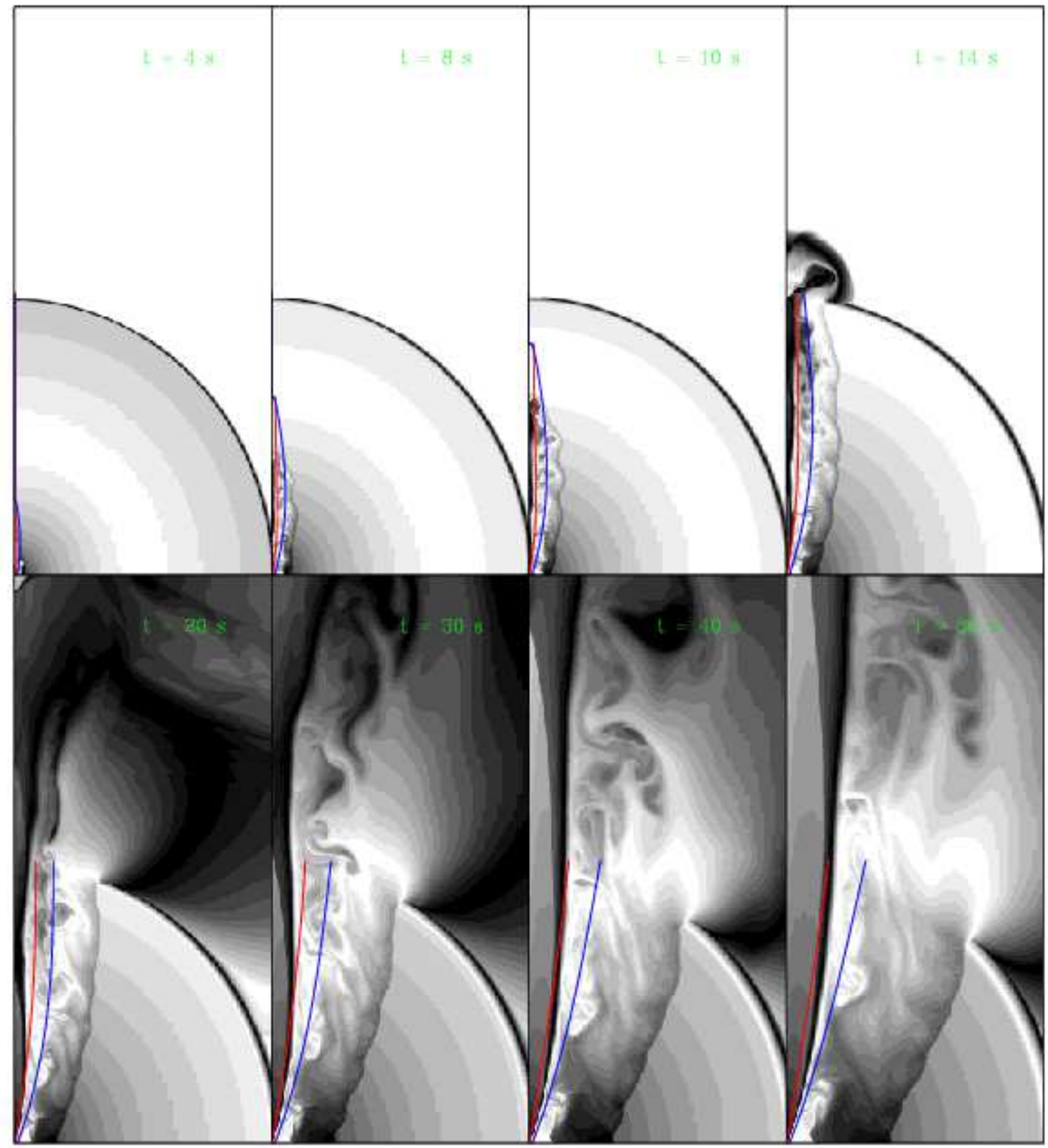

Fig. 18. - Comparison between the analytic and numerical results. The red (inner) line shows the jet boundary while the blue (outer) line shows the cocoon boundary. The simulations stills are from model t10g5. 

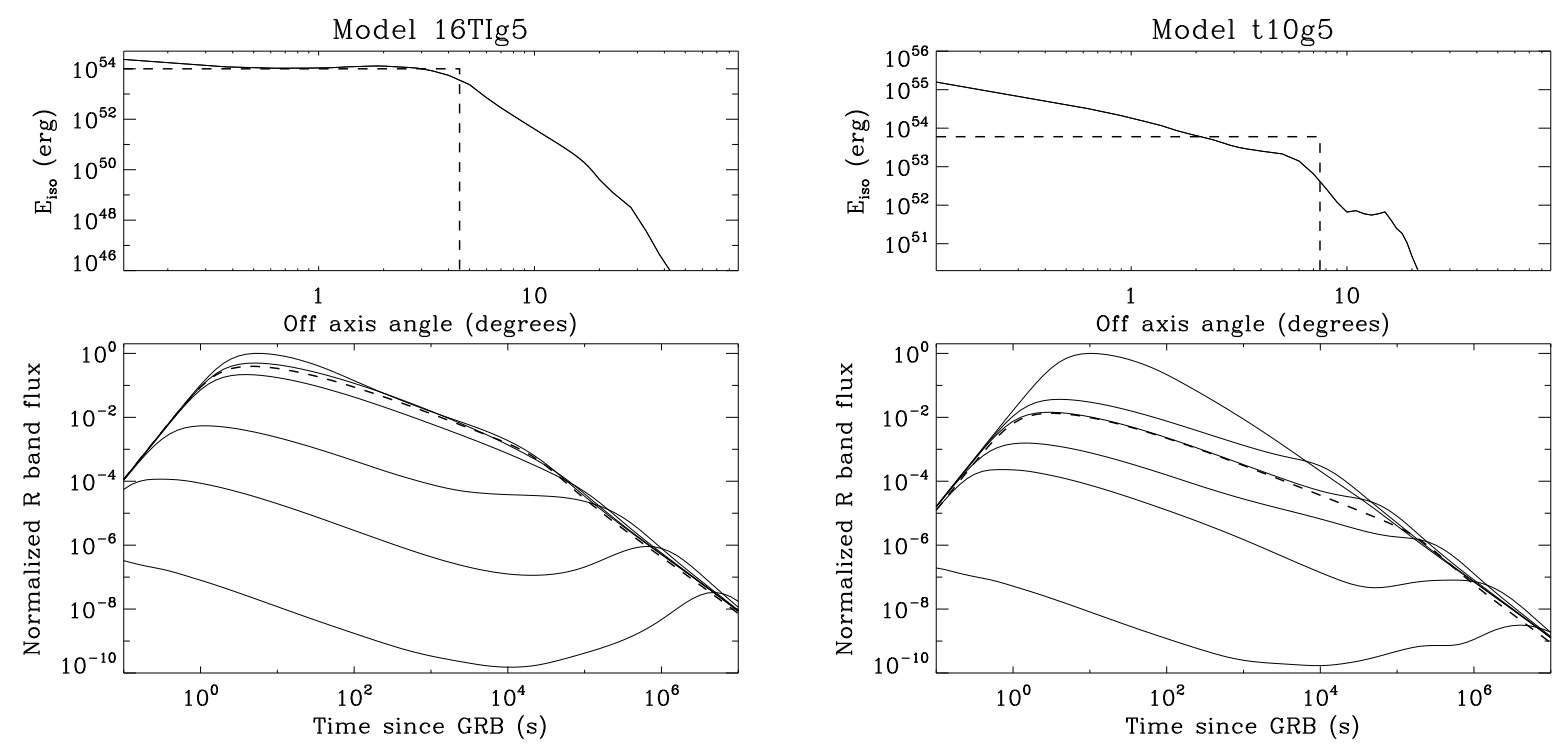

Fig. 19. - Afterglow light curves in the $\mathrm{R}$ band for the energy distribution obtained in model 16TIg5 (left panel) and t10g5 (right panel). Each panel shows the energy distribution in the top and the afterglow light curves on the bottom. Viewing angles $\theta_{0}=0,2,4,8,16$, and 32 degrees off-axis are shown, from top to bottom. A dashed curve shows, for comparison purposes, the afterglow of a flat energy distribution with an opening angle of $4^{\circ}$ and $7^{\circ}$ for model $16 \mathrm{TIg} 5$ and $\operatorname{t10g} 5$, respectively. All light curves have been normalized to the maximum of the on-axis light curve. 


\section{A. Code Testing}

In order to evaluate the accuracy of the FLASH relativistic hydrodynamics module, we have carried out a series of tests and compared the results to analytic solutions where possible. These results have also been compared to the weighted essentially non-oscillatory (WENO) scheme used by Zhang \& MacFadyen (2006) in their Relativistic Adaptive Mesh code (RAM hereafter). All test problems were run using FLASH's Riemann-type solver along with a piecewise-parabolic reconstruction scheme, identical to that used for the simulations presented in this paper. Although this scheme is formally less accurate and more diffusive than that used in RAM, our testing shows that the errors produced by FLASH are similar to those produced by RAM.

\section{A.1. 1D Riemann Problem 1}

For the 1D Riemann problem, there is a discontinuity between two states at $x=0.5$ on a grid ranging from $x=0$ to 1 . The values of pressure, density, perpendicular $\left(v_{x}\right)$ and transverse $\left(v_{y}\right)$ velocity can be different for the left and right states. For the first Riemann problem considered, the left state has a pressure of 13.33 and density of 10 while the right state has a pressure of $10^{-8}$ and density of 1 . The $x$ and $y$ velocity in both states is initially 0 . For this problem an adiabatic index of $\Gamma=5 / 3$ and CFL number of 0.5 are used. As time progresses, a mildly relativistic shock wave forms traveling to the right and a rarefaction wave travels to the left. The result at $t=0.4$ is then compared to the analytic solution to the identical problem (Pons et al. 2000), and the L1 error calculated. The L1 error is defined as $L 1=\Sigma_{j} \Delta x_{j}\left|u_{j}-u\left(x_{j}\right)\right|$ where $x_{j}$ is the coordinate of grid point $j$ and $u\left(x_{j}\right)$ and $u_{j}$ are the analytic and numerical values of proper density at grid point $j$ and $\Delta x_{j}$ is the grid spacing. This is identical to the definition of L1 error in RAM. Figure 20 shows a comparison between the numerical and analytic solutions at a resolution of 400 grid points. Table 4 lists the L1 errors and convergence rates for resolutions from 100 to 3200 grid points for this model, and compares it with the error and convergence rate from RAM. Convergence rate is defines as $\left[\ln \left(L 1_{i / 2} / L 1_{i}\right)\right] /[\ln 2]$ where $L 1_{i}$ is the $L 1$ error at resolution $i$.

For Riemann type problems, the L1 error is dominated by the few points near the shock front. This limits the convergence rate of any code to about 1 . It also makes the L1 error sensitive to the particular slope limiter being used. Therefore, small differences in the L1 error should not be taken to indicate a difference in the fundamental accuracy achievable by the two codes.

\section{A.2. 1D Riemann Problem 2}

For the second Riemann test, pressure is set to 1000 in the left state and $10^{-2}$ in the right state. Density in both states in initially 1 . The velocities in both states are again set to 0 with an adiabatic index of $5 / 3$ and CFL number of 0.5 . These conditions give rise to higher velocities than 
Riemann problem 1 and a narrower shock. Figure 21 shows a comparison between the numerical and analytic solutions at a resolution of 400 grid points at $t=0.4$. Table 5 lists the L1 errors and convergence rates for resolutions from 100 to 3200 grid points for this model, and compares it with the error and convergence rate from RAM.

\section{A.3. 1D Riemann Problem 3}

For the third standard Riemann test problem, a situation is set up in which a strong reverse shock develops. Pressure is set to 1 in the left state and 10 in the right state. Density in both states in initially 1 . Velocity in the $x$ direction is set to 0.9 in the left state and 0 in the right state. Transverse velocity $\left(v_{y}\right)$ is 0 in both states. An ultra-relativistic adiabatic index $(\Gamma=4 / 3)$ is used in this test along with a CFL number of 0.5. Figure 22 shows a comparison between the numerical and analytic solutions at a resolution of 400 grid points at $t=0.4$. Oscillations in the post-shock density of $\sim 1 \%$ are visible. Table 6 lists the L1 errors and convergence rates for resolutions from 100 to 3200 grid points for this model, and compares it with the error and convergence rate from RAM.

\section{A.4. 1D Riemann Problem 4: "Easy" Shear Velocity Test}

Analytical solutions can also be obtained for a 1D Riemann problem with a transverse (shear) velocity (Pons et al. 2000). For the "easy" version of this problem, the setup is identical to that of Riemann problem 2 above, but with a shear velocity of $v_{y}=0.99$ in the right state and $v_{y}=0$ in the left state. Figure 23 shows a comparison between the numerical and analytic solutions at a resolution of 400 grid points at $t=0.4$. Table 7 lists the L1 errors and convergence rates for resolutions from 100 to 3200 grid points for this model, and compares it with the error and convergence rate from RAM.

\section{A.5. 1D Riemann Problem 5: "Hard" Shear Velocity Test}

For the "hard" shear velocity test, the setup is again identical to that of Riemann problem 2 above, but with a shear velocity of $v_{y}=0.9$ in both the left and right states. This test is more difficult for numerical codes due to the shear velocity in the high-pressure left state. As the numerical solution poorly matches the analytic solution at low resolution, this test is run on higher resolution grids. Figure 24 shows a comparison between the numerical and analytic solutions at a resolution of 400 grid points at $t=0.6$. Table 8 lists the L1 errors and convergence rates for resolutions from 100 to 6400 grid points for this model, and compares it with the error and convergence rate from RAM. It should be noted that for the last 3 Riemann problems the absolute error using FLASH is significantly small than for the F-WENO scheme in RAM. In particular, 
comparing figure 24 to figure 9 in RAM it is apparent that FLASH produces a better fit to the analytic solution at low resolution. This is likely due to the different slope limiters being used by the two codes rather than fundamental limitations of the relativistic solvers they employ.

\section{A.6. 1D Isentropic Flow}

For all the Riemann problems, the error is dominated by the region near the shock, which gives approximately first order convergence regardless of the scheme used. To examine the behavior of the code in smooth flow regions, we evaluate an isentropic smooth flow set up in a uniform reference state. The initial density for this problem is given by

$$
\rho_{0}(x)=\rho_{\text {ref }}(1+\alpha f(x)),
$$

where $\rho_{\text {ref }}$ is the density of the reference state and

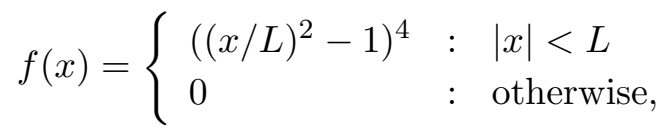

$\alpha$ and $L$ are the amplitude and width of the pulse. Pressure is given by the adiabatic equation of state $p=K \rho^{\Gamma}$, where $K$ is a constant. The initial velocity in the reference state $(|x|>L)$ is 0 . The initial velocity inside the pulse is found by assuming that one of the two Riemann invariants is constant,

$$
J_{-}=\frac{1}{2} \ln \left(\frac{1+v}{1-v}\right)-\frac{1}{\sqrt{\Gamma-1}} \ln \left(\frac{\sqrt{\Gamma-1}+c_{s}}{\sqrt{\Gamma-1}-c_{s}}\right)=\text { const }
$$

where

$$
c_{s}=\sqrt{\Gamma \frac{p}{\rho+\frac{\Gamma}{\Gamma-1} p}}
$$

is the sound speed. Solving for $v$ gives

$$
v=\frac{e^{2\left(J_{-}+\frac{1}{\sqrt{\Gamma-1}} \ln \left(\frac{\sqrt{\Gamma-1}+c_{s}}{\sqrt{\Gamma-1}-c_{s}}\right)\right.}-1}{e^{2\left(J_{-}+\frac{1}{\sqrt{\Gamma-1}} \ln \left(\frac{\sqrt{\Gamma-1}+c_{s}}{\sqrt{\Gamma-1}-c_{s}}\right)\right.}+1}
$$

where $J_{-}$is a constant calculated from the reference state. For the $1 \mathrm{D}$ isentropic test, our computational domain extends from $x=-0.35$ to $x=1.0$. The reference state is set to $\rho_{\text {ref }}=1$, $v_{\text {ref }}=0, p_{\text {ref }}=100, K=100$, with the pulse amplitude $\alpha=1.0$ and pulse length $L=0.3$. An adiabatic index of $\Gamma=5 / 3$ and a CFL number of 0.5 are used. The test is run until $t=0.8$, which is before a shock develops in the flow. An analytic solution for the density at this time can be found 
by characteristic analysis. A comparison of numerical and analytic results for density at $t=0.8$ is shown in figure 25. L1 errors and convergence rates for resolutions from 80 to 5120 grid points are shown in Tab. 9. This table shows that FLASH has a convergence rate of 2 for smooth flows, as expected for the solver being used (formally 2nd order accurate in time and space). The F-WENO scheme used in RAM has a convergence rate of $\sim 3$, also as expected (formally 5 th order accurate in space, 3rd order accurate in time). RAM is clearly more accurate in smooth flow regions, although both codes converge toward the correct solution.

\section{A.7. 2D Isentropic Flow}

The isentropic flow problem can also be used to test the behavior of the FLASH code in a two dimensional situation. For this test, the computational region goes from $0.0 \leq x \leq 3.75$ and $0.0 \leq y \leq 5.0$ in 2D Cartesian coordinates. The boundary conditions of the grid are periodic. Periodic isentropic waves with a spacing of 3.0 are place in the grid such that $\mathbf{k}$, the normal vector perpendicular to the wave front, is $\mathbf{k}=(4 / 5,3 / 5)$. Note that the spatial periods in the $x$ and $y$ direction are 3.75 and 5.0, so that periodic boundary conditions are appropriate. The initial density profile is given by $\rho_{0}(d)$ (eqn. A1) where $d=\bmod (\mathbf{k} \cdot \mathbf{r}+S / 2, S)-S / 2$ and $\mathbf{r}=(x, y)$. The reference state is set to $\rho_{\text {ref }}=1, v_{r e f}=0, p_{\text {ref }}=100, K=100$, with the pulse amplitude $\alpha=1.0$ and pulse length $L=0.9$. An adiabatic index of $\Gamma=5 / 3$ and a CFL number of 0.5 are used. The test is run until $t=2.4$. Table 10 shows the total L1 errors and convergence rates for grid resolutions from $48 \times 64$ to $768 \times 1024$ grid points. The convergence rate for FLASH is again about 2 , as expected.

The above results confirm that the relativistic FLASH module used here converges to the correct solution for problems with known analytic results and has a comparable level of accuracy to other available relativistic hydro codes.

\section{A.8. 2D Riemann Problem}

The Riemann problem can be extended to two dimensions by setting up a square grid in which each quarter has different initial conditions. Although there is no analytic solution to this problem, it has been well studied as a test of relativistic codes (Del Zanna \& Bucciantini 2002; Lucas-Serrano et al. 2004; Zhang \& MacFadyen 2006). The computational domain extends from $0.0 \leq x \leq 1.0$

and $0.0 \leq y \leq 1.0$ in $2 \mathrm{D}$ Cartesian coordinates. The initial conditions for the four quarters of the grid are

$$
\begin{array}{llll}
\left(\rho, v_{x}, v_{y}, p\right)=(0.1,0,0,0.01) & \text { for } & 0.5 \leq x \leq 1.0 & 0.5 \leq y \leq 1.0 \\
\left(\rho, v_{x}, v_{y}, p\right)=(0.1,0.99,0,1 .) & \text { for } & 0.0 \leq x \leq 0.5 & 0.5 \leq y \leq 1.0 \\
\left(\rho, v_{x}, v_{y}, p\right)=(0.5,0,0,1 .) & \text { for } & 0.0 \leq x \leq 0.5 & 0.0 \leq y \leq 0.5 \\
\left(\rho, v_{x}, v_{y}, p\right)=(0.1,0,0.99,1 .) & \text { for } & 0.5 \leq x \leq 1.0 & 0.0 \leq y \leq 0.5
\end{array}
$$


An adiabatic index of $\Gamma=5 / 3$ is used with a CFL number of 0.2 on a grid of $512 \times 512$ grid points. Figure 26] shows logarithmically spaced density contours of the test at $t=0.4$. Our test correctly reproduces the two curved shock fronts and sharp density spike in the upper right portion of the grid seen in other codes. The low density flow moving toward the lower left is also seen, although it appears more turbulent than in other codes. Symmetry is not perfectly preserved across the diagonal from lower left to upper right. This is because FLASH uses operator splitting rather than a Runge-Kutta integration scheme such as that used in RAM. However, this loss of symmetry is not important as our code is being used to examine jets in $2 \mathrm{D}$ cylindrical coordinates where there is no symmetry to preserve.

\section{A.9. Isentropic Pulse}

To examine what happens when material crosses a change in grid refinement, we set up a 2D isentropic pulse in density only that is advected across changes in refinement and eventually returned to its original location. For this problem the computational domain ranges from $0.0 \leq x \leq 0.9$ and $0.0 \leq y \leq 0.9$ in $2 \mathrm{D}$ Cartesian coordinates with periodic boundaries. The resolution of the mesh varies from $\Delta x=0.0225$ at the edges to $\Delta x=0.0028125$ in the innermost portion of the grid (see figure 27 for block structure). The structure of the grid is fixed and does not change as the density pulse moves. The pulse in initially centered at $x=0.45, y=0.45$ and is set by eqn. A1 with $\alpha=10$ and $L=0.2$. Velocity everywhere is set to $v_{x}=0.72$ and $v_{y}=0.54$, for a total velocity of $v=0.9$. Pressure everywhere is $p=1$, the adiabatic index is $\Gamma=5 / 3$ and the CFL number is 0.5. The test is run until $t=10$, at which time the pulse is again centered at the center of the computational domain. As can be seen in figure 27, the density at the center of the pulse has been flattened and the shape has become somewhat more square due to changes in refinement as the pulse has advected. However, there are no spurious waves and the size of the pulse has not changed significantly. At $t=10$, no fluctuations in pressure are detectable. A comparison with figure 10 in RAM for an identical test shows that while their pulse has increased in width by $\sim 35 \%$, there has been almost no increase in width in our test, indicating that, in this case, FLASH has less numerical diffusion.

\section{A.10. Emery Step}

The Emery step problem (Emery 1968; Woodward \& Colella 1984) consists of a wind flowing into a sharp vertical step in a wind tunnel with reflective upper and lower boundaries. The step causes a reverse shock to propagate into the wind. The shock will eventually collide with the upper boundary and reflect, giving rise to a Mach stem which is initially nearly vertical. By the end of the test a portion of the reflected shock has again reflected, this time off the lower boundary. For this test, a computational domain ranging from $0.0 \leq x \leq 3.0$ and $0.0 \leq y \leq 1.0$ in 2D Cartesian coordinates is used with a step of height 0.2 beginning at $x=0.6$. Initially, the grid is filled with 
gas of $\rho=1.4$ and $v=0.999$ with an adiabatic index of $\Gamma=1.4$ moving with a Newtonian Mach number of 3.0. Using eqn. A4 gives a pressure of $p=0.1534$. Upper and lower boundaries, as well as the face of the step, are reflective. The left boundary is a constant inflow with these initial parameters. The right boundary uses outflow boundary conditions. A CFL number of 0.5 is used. The test is run to a time of $t=4$. Figure 28 shows contours of density for the results carried out on a uniform grid of $240 \times 80$ grid points. Our results have very little noise in the downstream region and along the top of the step, as compared to the U-PPM results in RAM, which employ a similar solver to FLASH (see RAM figure 7). Our results appear more comparable to the F-WENO method in RAM.

This test has also been carried out on an adaptive mesh grid with 5 levels of refinement, for a maximum resolution of $3840 \times 1280$ grid points. Other than the resolution, the setup is identical to that of the uniform grid. As this problem involves mostly smooth flows, it provides a good test of the ability of FLASH to selectively refine and de-refine while still capturing shocks and discontinuities. Logarithmically spaced density contours of the results at $t=4$ are shown in the lower frame of figure 28. The density and pressure are plotted in figure 29 along with the velocity field and block structure of the mesh. The figure is comparable to figure 15 from RAM, which shows the same results for an identical setup and maximum resolution. Both codes concentrate refinement around the shocks that develop and the contact discontinuity that originates from the bottom of the Mach stem. Using our code, Kelvin-Helmholtz instabilities are clearly seen along this contact discontinuity. This instability does not develop using RAM, which may indicate that FLASH is less diffusive.

\section{A.11. Double Mach Reflection}

The double Mach reflection test presented here follows the same setup as in RAM. This test consists of a computational domain ranging from $0.0 \leq x \leq 4.0$ and $0.0 \leq y \leq 1.0$ in $2 \mathrm{D}$ Cartesian coordinates. A shock is placed on the grid moving down and to the right at a 60 degree angle to the $\mathrm{x}$ axis. The lower boundary is reflecting for $x>1 / 6$. At the initial time, the shock is just making contact with the reflecting portion of the lower boundary. The lower boundary for $x \leq 1 / 6$ is set to the post-shock conditions, as is the left boundary. The upper boundary is set to either the pre- or post-shock conditions depending on the time of the simulation. The right boundary is always set to the pre-shock conditions for the test considered here. The unshocked conditions are $\rho_{0}=1.4$, $p_{0}=0.0025$, and $v_{0}=0.0$ with an adiabatic index of $\Gamma=1.4$ in both the pre- and post-shock state. The shock is moving with a classical Mach number of $M=v_{s} / c_{s}$ of 10 where $v_{s}$ is the shock speed and $c_{s}$ is the sound speed of the unshocked gas. The relativistic shock jump conditions can then be used to determine the post-shock conditions and the shock speed. Using equation A4, we find that $v_{s}=10 \times c_{s}=0.4984$ in the observer's frame. In the shock frame, this is the velocity of the incoming unshocked gas. Solving the relativistic shock jump conditions yields a post-shock state of

$\rho_{1}=8.564, p_{1}=0.3808, v_{1}=0.09358$ in the shock frame. In the observer frame this transforms to 
$v_{1}=0.4247$. The speed of the leading edge of the shock is the relativistic sum of the shock speed, $v_{s}$, and the sound speed of the shocked gas, which is $c_{s 1}=0.2321$, giving a total speed of 0.5978 for the leading edge of the shock. This is the velocity used to determine if a point on the upper boundary is in the pre- or post-shock state. A CFL number of 0.5 is used. The test is run to a time of $t=4$.

Figure 30 shows density contours of this test run on a uniform grid of $512 \times 128$ grid points and an adaptive mesh grid with the same maximum resolution. The contours of the two plots are nearly identical whether or not adaptive mesh is used. Contours produced using FLASH do not appear to be as smooth as those from RAM (see RAM figure 16), particularly to the left of the vertical shock at $x=2.7$. This may indicate that RAM produces more accurate results for this test.

\section{A.12. Spherical Implosion in Cylindrical Coordinates}

In order to test the behavior of the FLASH code in cylindrical coordinates we have carried out tests with spherical implosions and explosions. The spherical implosion problem consists of a spherically symmetric flow converging on a single point. This test is carried out on a computational domain of $0.0 \leq r \leq 1.0$ and $0.0 \leq z \leq 1.0$ in $2 \mathrm{D}$ cylindrical coordinates. Initially, $\rho_{0}=1$ and $p=0$ everywhere and the material is flowing toward the origin at a fixed speed $v$, and the adiabatic index is $\Gamma=4 / 3$. The $r=0$ and $z=0$ boundaries are reflecting and the $r=1$ and $z=1$ boundaries are set to the analytic solution for a spherically converging flow for the time in the simulation (Martí et al. 1997). The analytic solution used at the boundaries is

$$
\rho(r, t)=\rho_{0}\left(1+\frac{|v| t}{r}\right)^{2}
$$

where $r$ is the spherical radius, $t$ is the simulation time, and $v$ is the inflow velocity. Pressure at the boundaries is always 0 and $v$ is fixed. The converging flow forms a spherical region of shocked, stationary gas which increases in radius with time according to

$$
R_{s}=\frac{(\Gamma-1) \gamma|v|}{\gamma+1} t
$$

where $\gamma$ is the Lorentz factor of the inflowing gas. The density in the post-shock state is given by

$$
\rho_{s}=\rho\left(R_{s}, t\right)\left(\frac{\gamma \Gamma+1}{\Gamma-1}\right)
$$

This test was run for inflow velocities of $v=0.9, v=0.999$, and $v=0.99999$ to a time of $t=2$. All test were run with a CFL number of 0.2 . For these three tests, the average error in density in the post-shock gas is $3.16 \%, 1.91 \%$, and $8.37 \%$, respectively. Decreasing the CFL number will decrease the error, but as this is generally a hard test problem, these errors are acceptable. 


\section{A.13. Spherical Explosion in Cylindrical Coordinates}

For the spherical explosion test, the computational domain ranges from $0.0 \leq r \leq 1.0$ and $0.0 \leq z \leq 1.0$ in $2 \mathrm{D}$ cylindrical coordinates. Inside a radius of $R=0.4$ from the origin there is initially a gas with $\rho=1, p=1000$, and $v=0$ while outside this radius the gas has $\rho=1, p=1$, and $v=0$. The adiabatic index is $\Gamma=5 / 3$ in both states. This pressure discontinuity gives rise to a spherical shock traveling outward and a rarefaction wave traveling inward. Our test was carried out on a adaptive mesh grid with 4 levels of refinement for a maximum resolution of $320 \times 320$ grid points. The test was run with a CFL number of 0.2 to a time of $t=0.4$. The $r=0$ and $z=0$ boundaries are reflecting and the $r=1$ and $z=1$ boundaries allow free outflow. Figure 31 shows the density vs. (spherical) radius for points along the line $r=z$. Our results are consistent with results from other codes. The shock front is resolved by $\sim 4$ grid points. 
Table 4. 1D Riemann Problem 1

\begin{tabular}{ccccc}
\hline \hline Grid Points & FLASH L1 error & FLASH convergence rate & WENO L1 error & WENO convergence rate \\
\hline 100 & 0.132 & $\ldots$ & 0.131 & $\ldots$ \\
200 & 0.0696 & 0.92 & 0.0725 & 0.85 \\
400 & 0.0357 & 0.96 & 0.0332 & 1.1 \\
800 & 0.0179 & 1.0 & 0.0208 & 0.67 \\
1600 & 0.00852 & 1.1 & 0.0100 & 1.1 \\
3200 & 0.00432 & 0.98 & 0.00507 & 0.98 \\
\hline
\end{tabular}

Table 5. 1D Riemann Problem 2

\begin{tabular}{ccccc}
\hline \hline Grid Points & FLASH L1 error & FLASH convergence rate & WENO L1 error & WENO convergence rate \\
\hline 100 & 0.206 & $\ldots$ & 0.210 & $\ldots$ \\
200 & 0.148 & 0.48 & 0.142 & 0.56 \\
400 & 0.0832 & 0.83 & 0.0929 & 0.61 \\
800 & 0.0461 & 0.85 & 0.0554 & 0.75 \\
1600 & 0.0249 & 0.89 & 0.0254 & 1.1 \\
3200 & 0.0130 & 0.94 & 0.0151 & 0.75 \\
\hline
\end{tabular}

Table 6. 1D Riemann Problem 3

\begin{tabular}{ccccc}
\hline \hline Grid Points & FLASH L1 error & FLASH convergence rate & WENO L1 error & WENO convergence rate \\
\hline 100 & 0.0587 & $\ldots$ & 0.0997 & $\ldots$ \\
200 & 0.0347 & 0.76 & 0.0629 & 0.67 \\
400 & 0.0214 & 0.70 & 0.0301 & 1.1 \\
800 & 0.0133 & 0.69 & 0.0169 & 0.83 \\
1600 & 0.00845 & 0.65 & 0.00948 & 0.83 \\
3200 & 0.00329 & 1.36 & 0.00524 & 0.86 \\
\hline
\end{tabular}


Table 7. 1D Riemann Problem 4: "Easy" Shear Velocity Test

\begin{tabular}{ccccc}
\hline \hline Grid Points & FLASH L1 error & FLASH convergence rate & WENO L1 error & WENO convergence rate \\
\hline 100 & 0.627 & $\ldots$ & 0.758 & $\ldots$ \\
200 & 0.337 & 0.90 & 0.392 & 0.95 \\
400 & 0.172 & 0.97 & 0.231 & 0.76 \\
800 & 0.0843 & 1.0 & 0.118 & 0.97 \\
1600 & 0.0441 & 0.93 & 0.0658 & 0.84 \\
3200 & 0.0232 & 0.93 & 0.0344 & 0.94 \\
\hline
\end{tabular}

Table 8. 1D Riemann Problem 5: "Hard" Shear Velocity Test

\begin{tabular}{ccccc}
\hline \hline Grid Points & FLASH L1 error & FLASH convergence rate & WENO L1 error & WENO convergence rate \\
\hline 100 & 0.512 & $\ldots$ & $\ldots$ & $\ldots$ \\
200 & 0.464 & 0.14 & $\ldots$ & $\ldots$ \\
400 & 0.325 & 0.51 & 0.521 & $\ldots$ \\
800 & 0.217 & 0.58 & 0.363 & 0.52 \\
1600 & 0.133 & 0.71 & 0.233 & 0.64 \\
3200 & 0.0833 & 0.68 & 0.126 & 0.89 \\
6400 & 0.0534 & 0.64 & 0.0649 & 0.96 \\
\hline
\end{tabular}

Table 9. 1D Isentropic Flow

\begin{tabular}{ccccc}
\hline \hline Grid Points & FLASH L1 error & FLASH convergence rate & WENO L1 error & WENO convergence rate \\
\hline 80 & $5.48 e-3$ & $\ldots$ & $2.07 e-3$ & $\ldots$ \\
160 & $1.55 e-3$ & 1.8 & $1.10 e-4$ & 4.2 \\
320 & $3.99 e-4$ & 2.0 & $1.70 e-5$ & 2.7 \\
640 & $1.00 e-4$ & 2.0 & $1.47 e-6$ & 3.5 \\
1280 & $2.50 e-4$ & 2.0 & $1.58 e-7$ & 3.2 \\
2560 & $5.35 e-6$ & 2.2 & $1.91 e-8$ & 3.1 \\
5120 & $1.56 e-6$ & 1.8 & $2.37 e-9$ & 3.0 \\
\hline
\end{tabular}


Table 10. 2D Isentropic Flow

\begin{tabular}{ccccc}
\hline \hline Grid Points & FLASH L1 error & FLASH convergence rate & WENO L1 error & WENO convergence rate \\
\hline $48 \times 64$ & $8.12 e-3$ & $\ldots$ & $7.35 e-2$ & $\ldots$ \\
$96 \times 128$ & $2.23 e-3$ & 1.9 & $4.43 e-3$ & 4.1 \\
$192 \times 256$ & $5.87 e-4$ & 1.9 & $8.04 e-4$ & 2.5 \\
$384 \times 512$ & $1.48 e-4$ & 2.0 & $9.62 e-5$ & 3.1 \\
$768 \times 1024$ & $3.61 e-5$ & 2.0 & $1.12 e-5$ & 3.1 \\
\hline
\end{tabular}




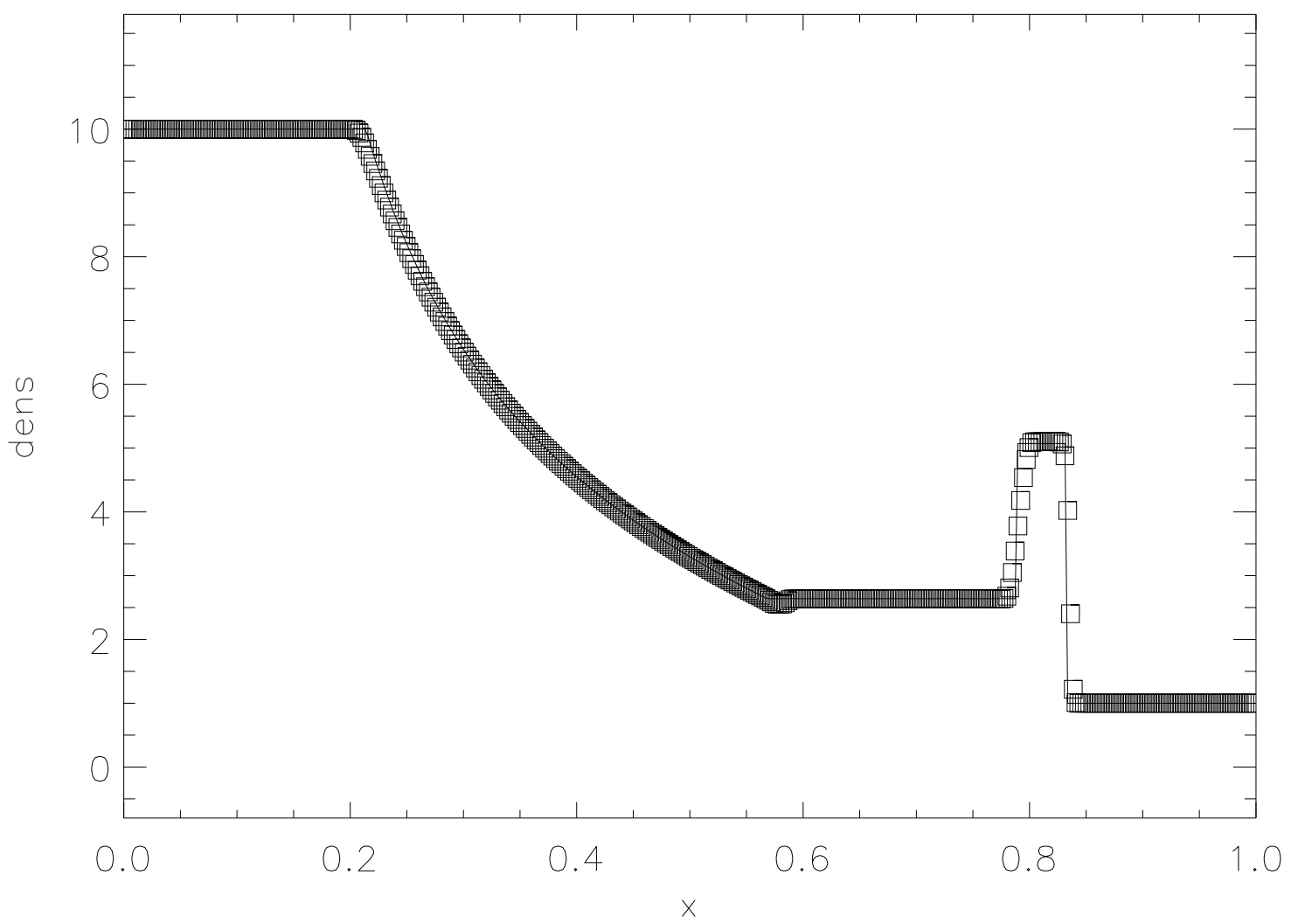

Fig. 20.- 1D Riemann Problem 1. Density for the analytic solution (solid line) and numerical solution (squares) with a resolution of 400 grid points is plotted at $t=0.4$. 


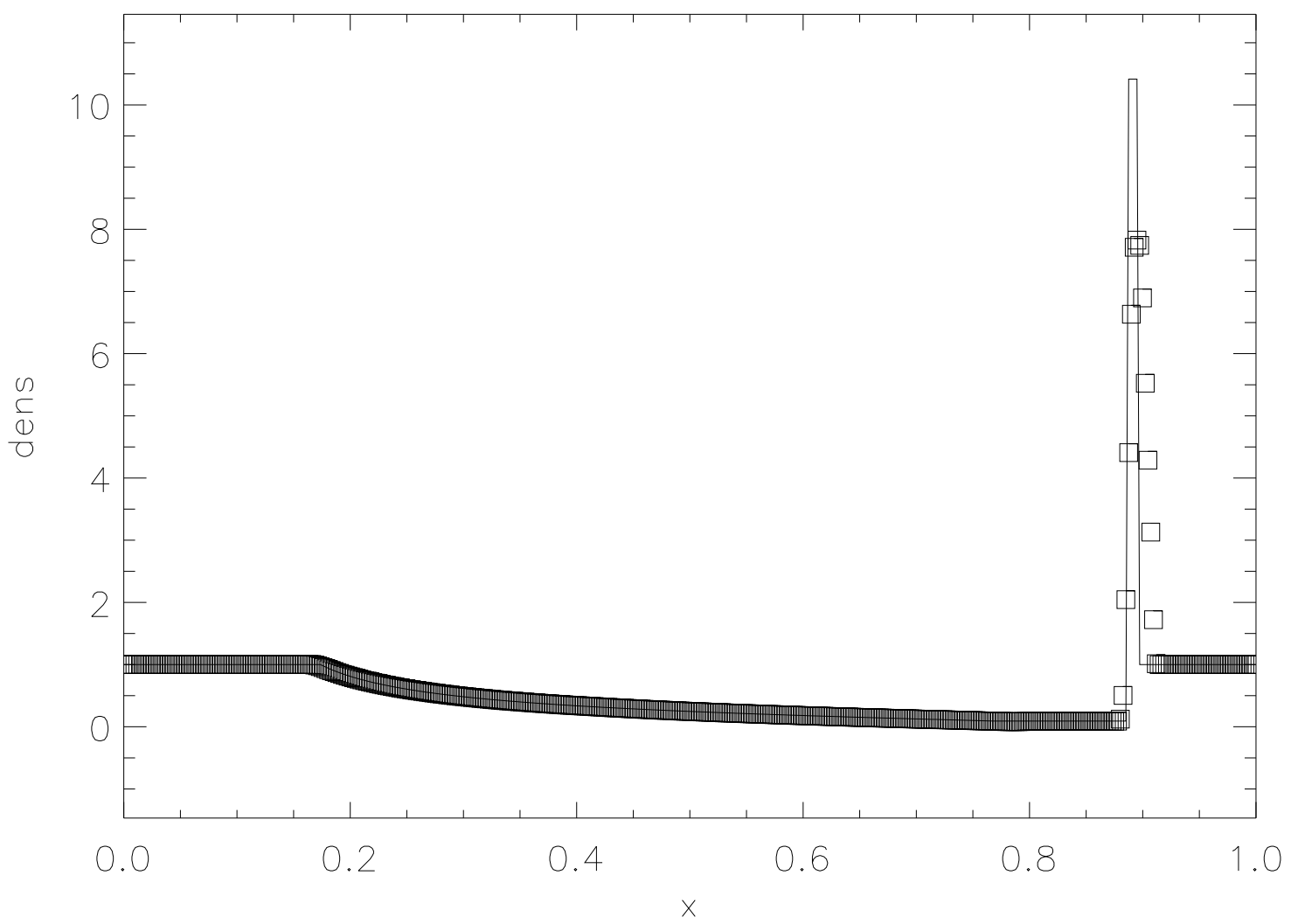

Fig. 21.- 1D Riemann Problem 2. Density for the analytic solution (solid line) and numerical solution (squares) with a resolution of 400 grid points is plotted at $t=0.4$. 


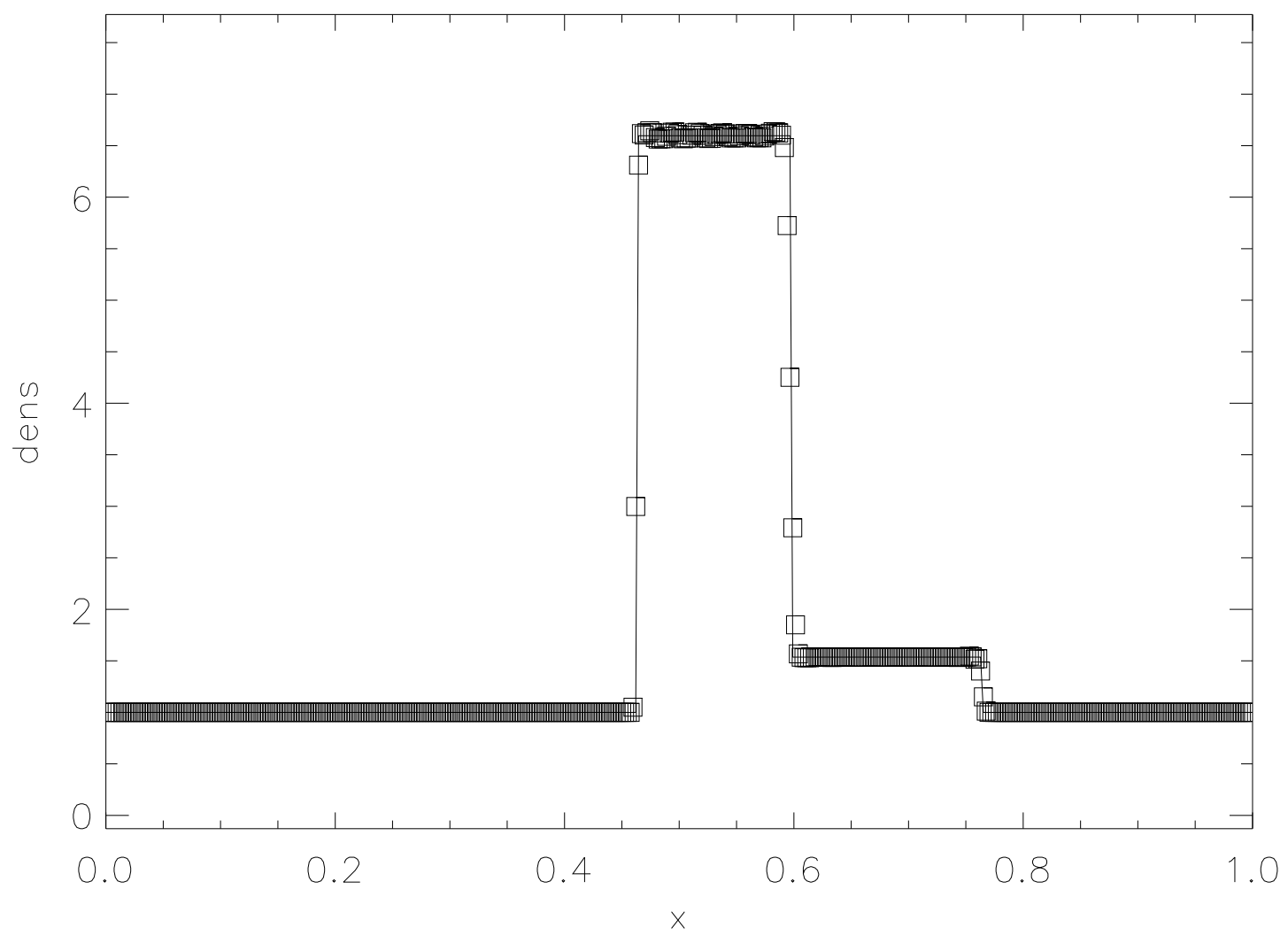

Fig. 22.- 1D Riemann Problem 3. Density for the analytic solution (solid line) and numerical solution (squares) with a resolution of 400 grid points is plotted at $t=0.4$. 


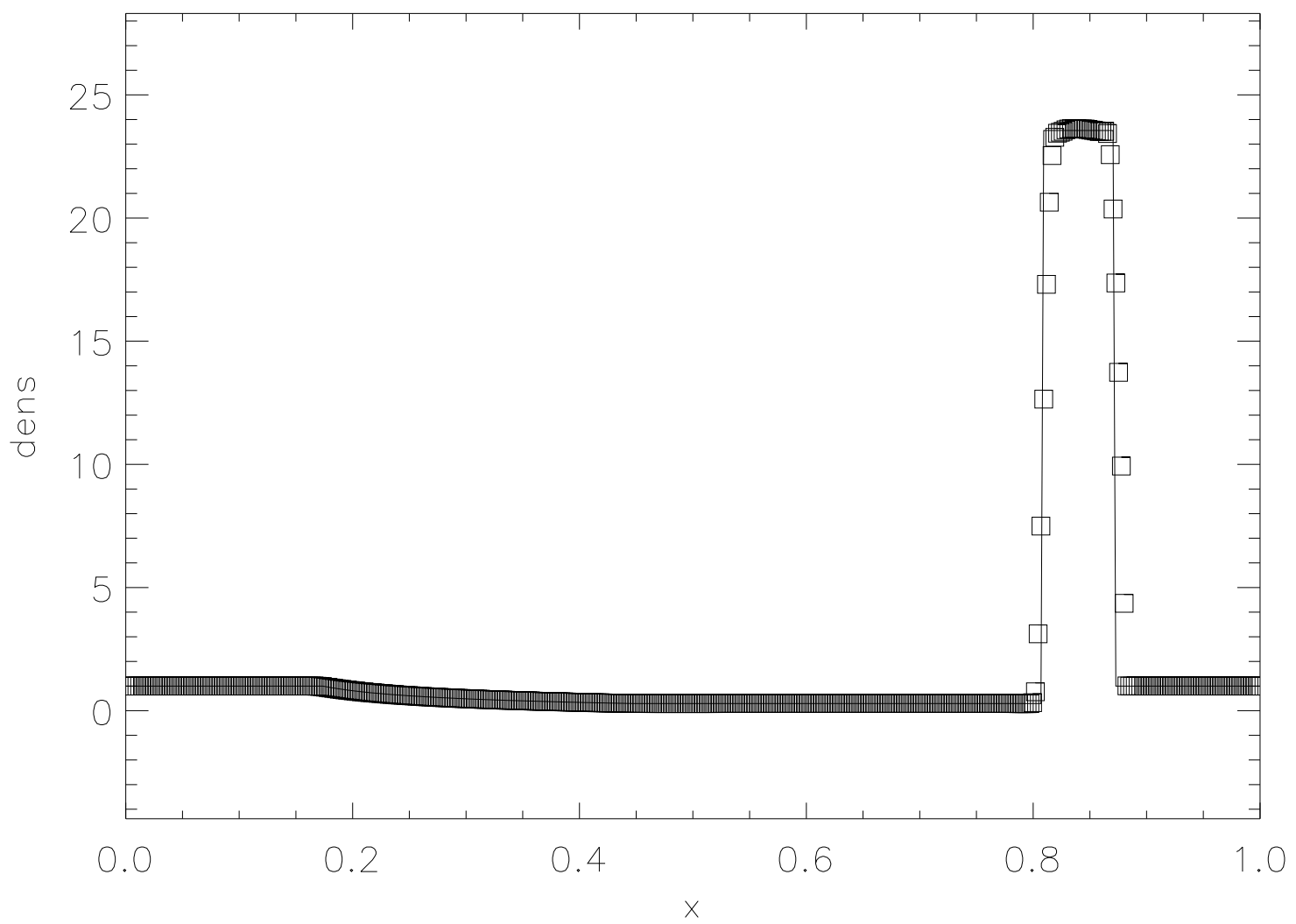

Fig. 23.- 1D Riemann Problem 4: "Easy" Shear Velocity Test. Density for the analytic solution (solid line) and numerical solution (squares) with a resolution of 400 grid points is plotted at $t=0.4$. 


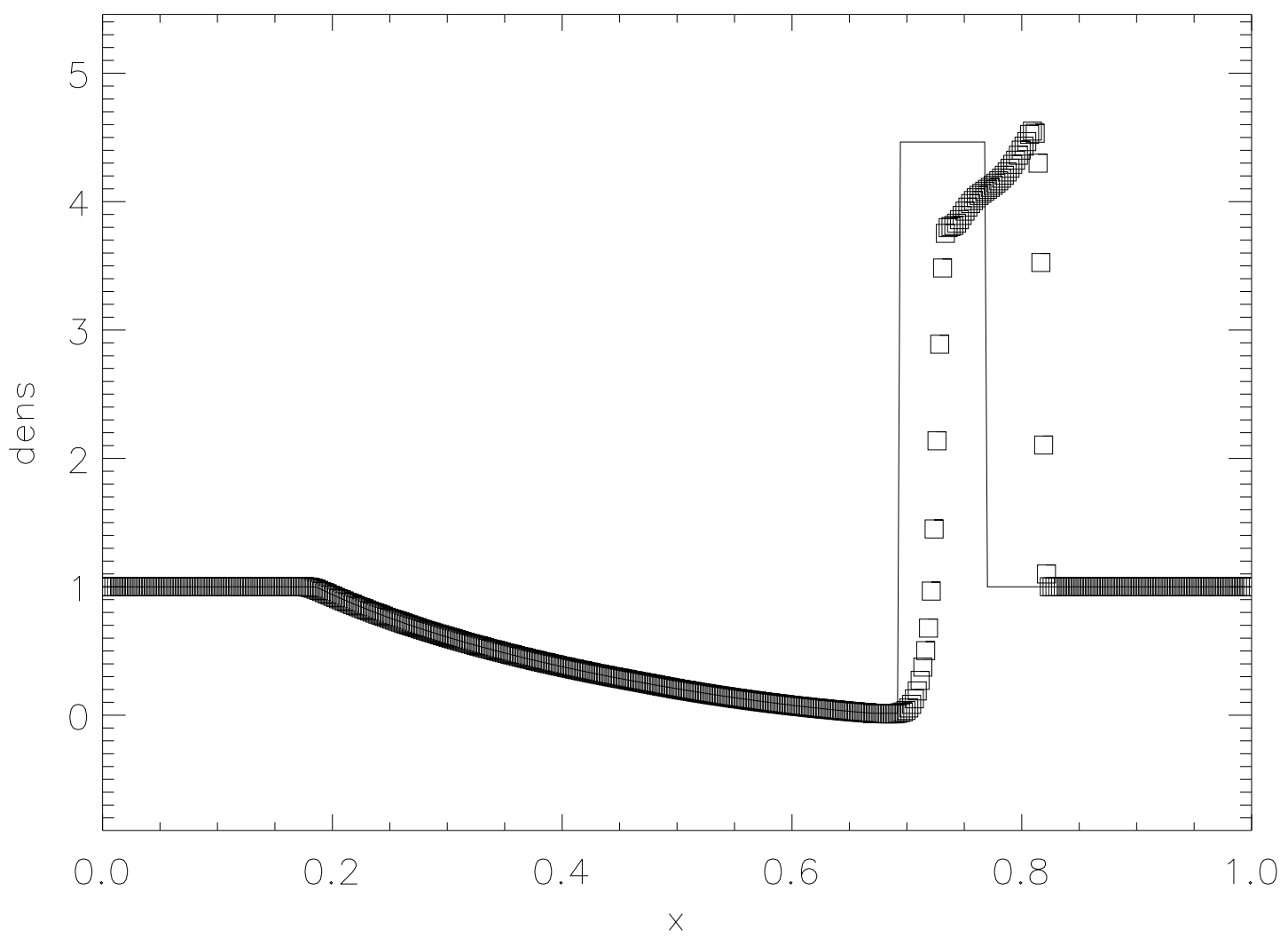

Fig. 24.- 1D Riemann Problem 5: "Hard" Shear Velocity Test. Density for the analytic solution (solid line) and numerical solution (squares) with a resolution of 400 grid points is plotted at $t=0.6$. 


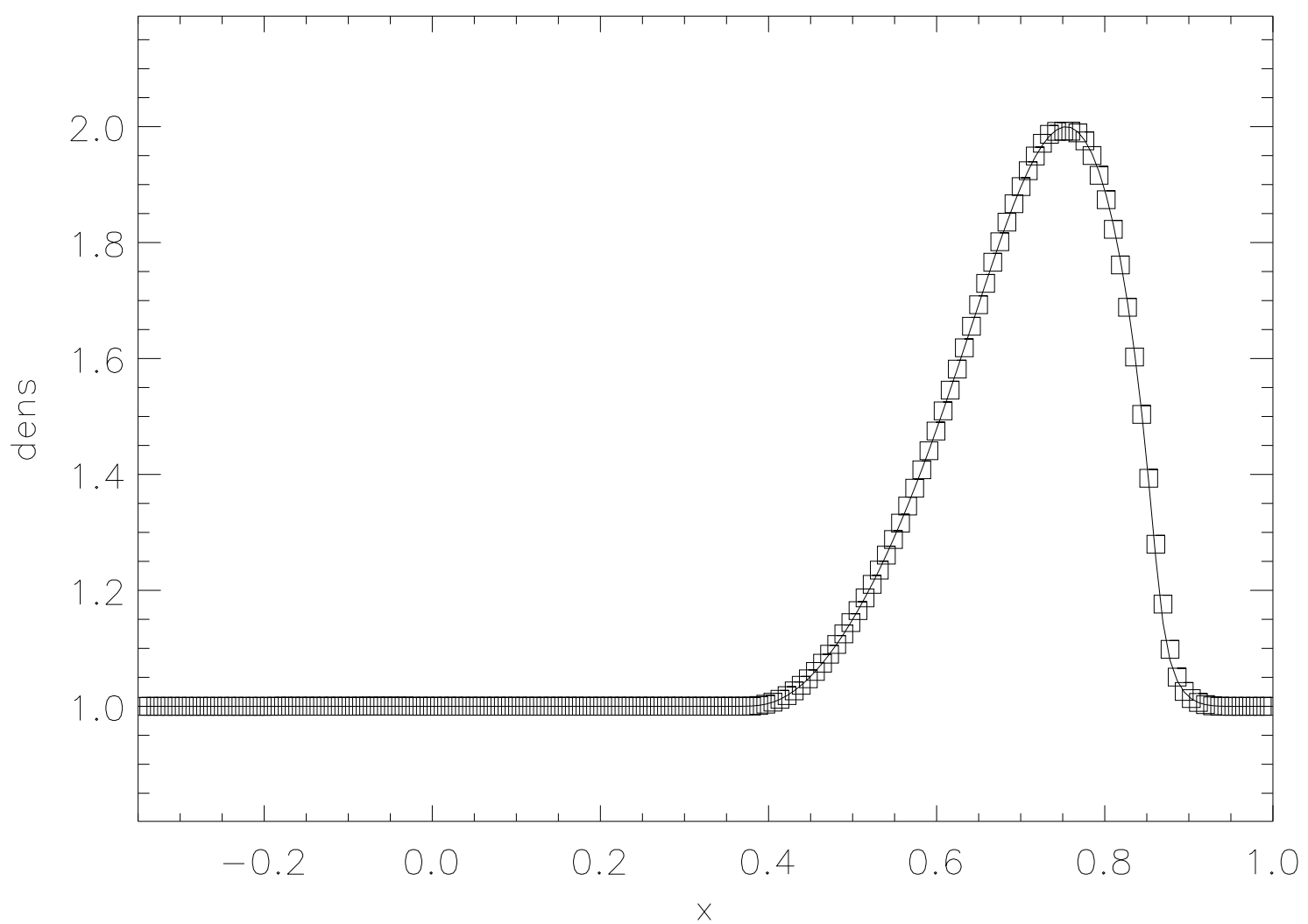

Fig. 25. - 1D isentropic flow. Density for the analytic solution (solid line) and numerical solution (squares) with a resolution of 160 grid points is plotted at $t=0.8$. 


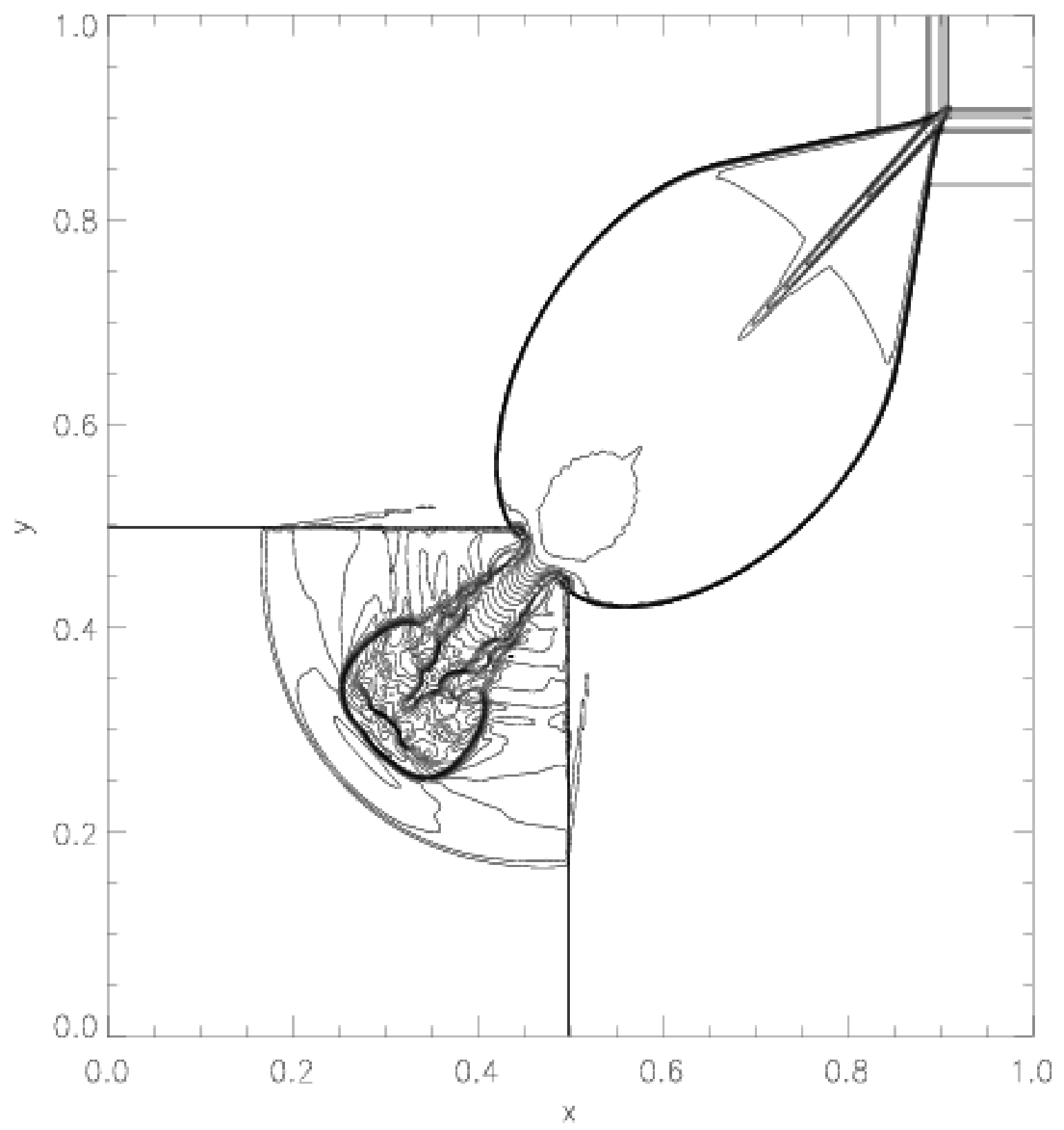

Fig. 26. $-2 \mathrm{D}$ Riemann problem at $t=0.4$. Thirty logarithmically spaced contours of density are plotted for a uniform grid of $512 \times 512$ grid points. 

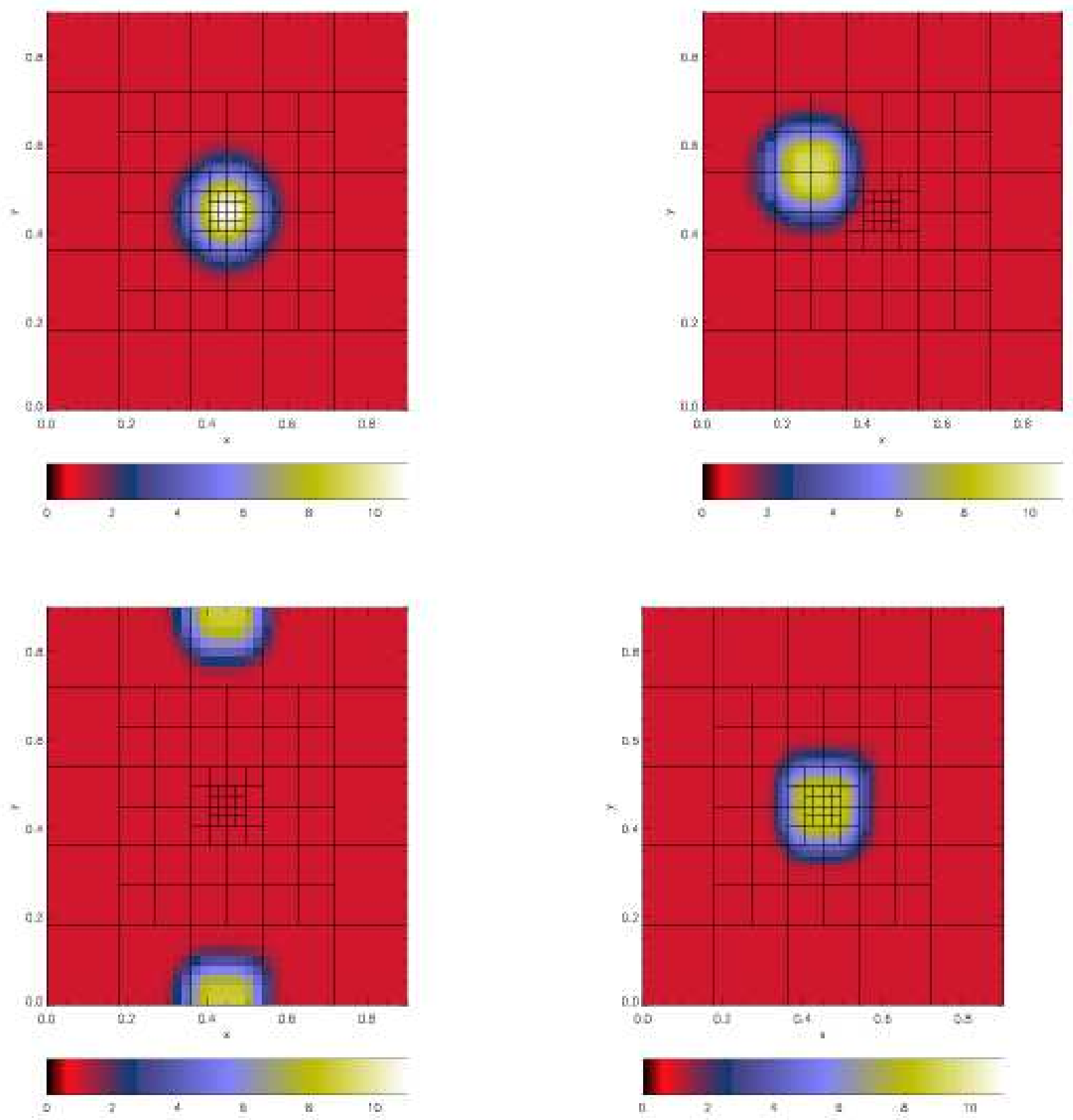

Fig. 27.- Isentropic pulse density at $t=0$ (upper left), 3.5 (upper right), 7.5 (lower left), and 10 (lower left). Block structure of the mesh is shown by black lines. Each block contains $8 \times 8$ grid points. The pulse moves up and to the right at a speed of $v=0.9$ at an angle of $\arctan (4 / 3)$. 

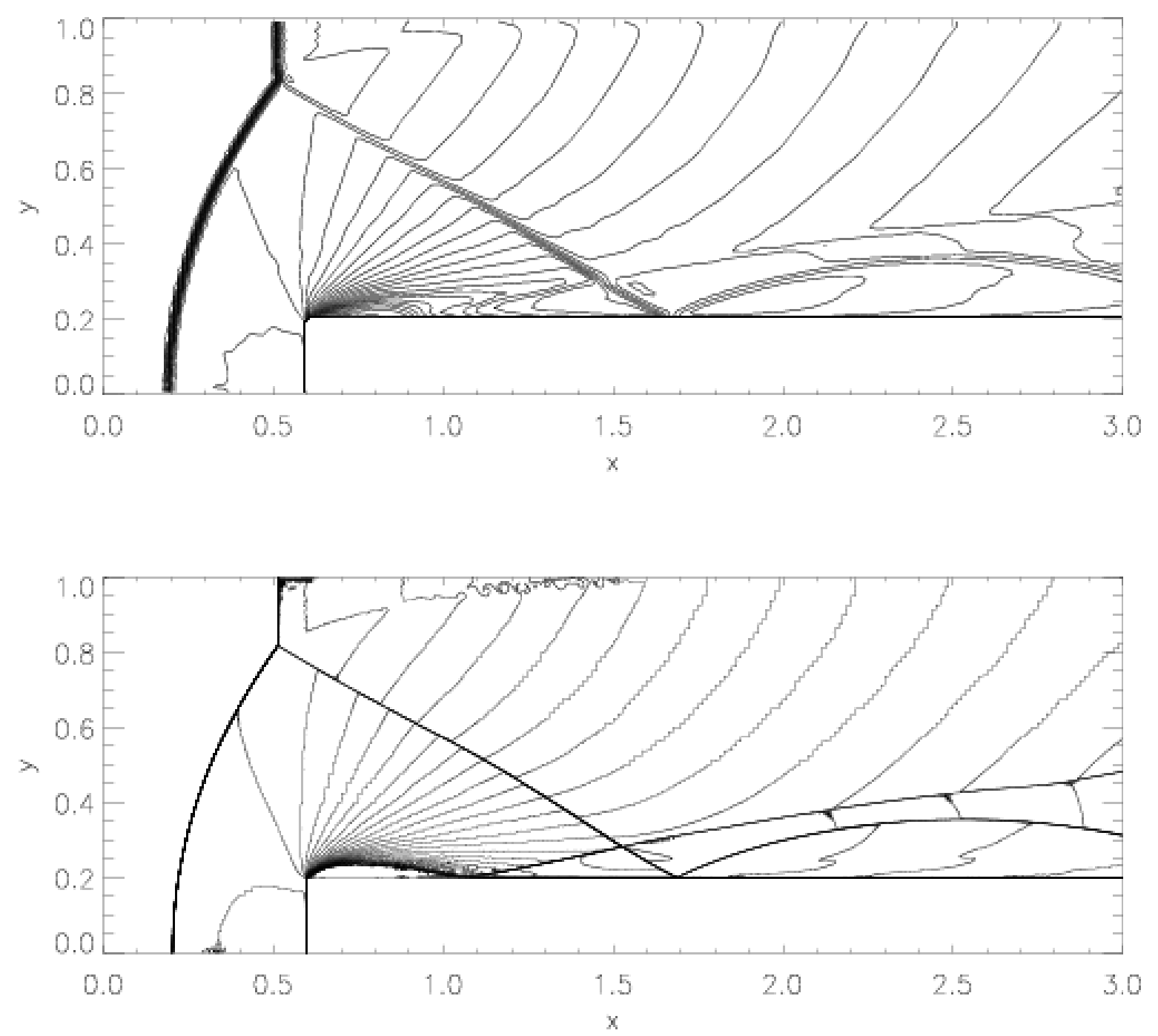

Fig. 28. - Emery step at $t=4$. Thirty logarithmically spaced contours of density are plotted for a uniform grid of $240 \times 80$ grid points (upper panel) and for an adaptive grid of up to $3840 \times 1280$ grid points. All the same features are apparent in the low resolution test, and there are no artifacts or instabilities in the smooth downstream region. Also note the development of a Kelvin-Helmholtz instability along the contact discontinuity originating from the vertical Mach stem in the lower panel. 

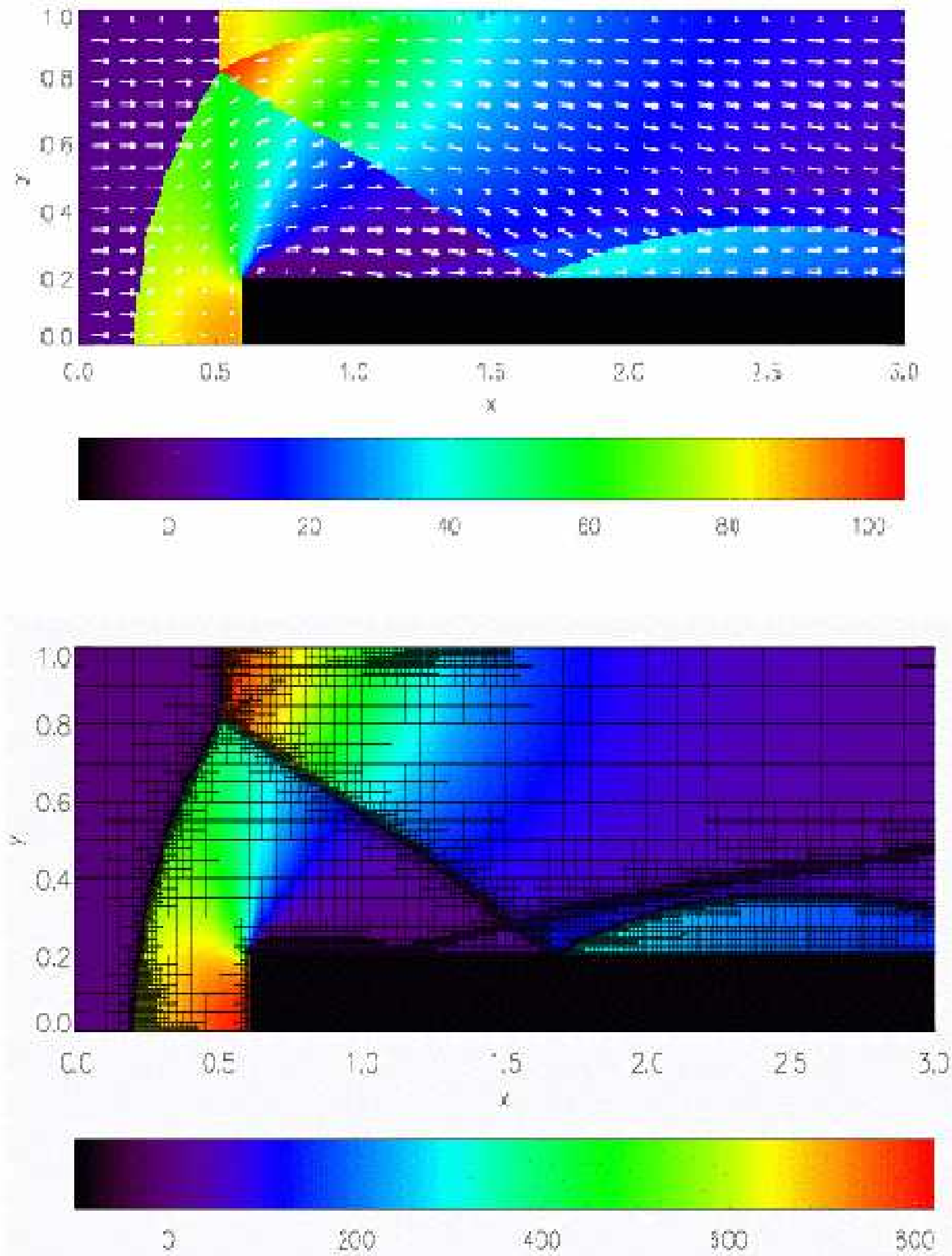

Fig. 29.- Emery step at $t=4$ for 5 level adaptive mesh (maximum $3840 \times 1280$ grid points). The upper panel shows (linear) density along with the velocity field (white arrows). The lower panel shows pressure along with the block structure of the adaptive grid (black lines). Each block 

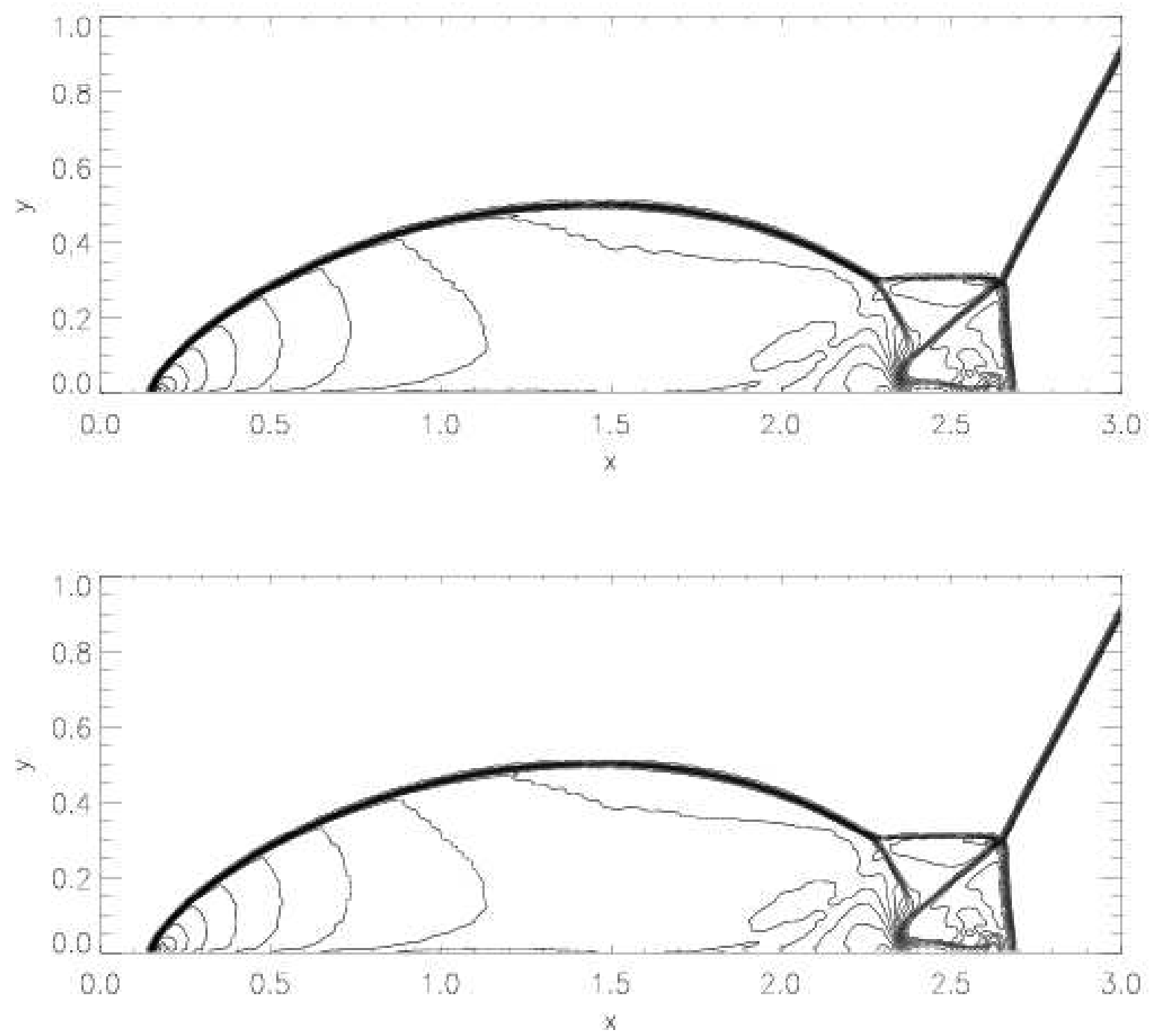

Fig. 30.- Double Mach reflection at $t=4$. Thirty equally spaced contours of density are plotted for a uniform grid of $512 \times 128$ grid points (upper panel) and an adaptive mesh grid with 4 levels of refinement with a maximum equivalent resolution of $512 \times 128$ grid points (lower panel). Note that the entire computational domain is not shown. 


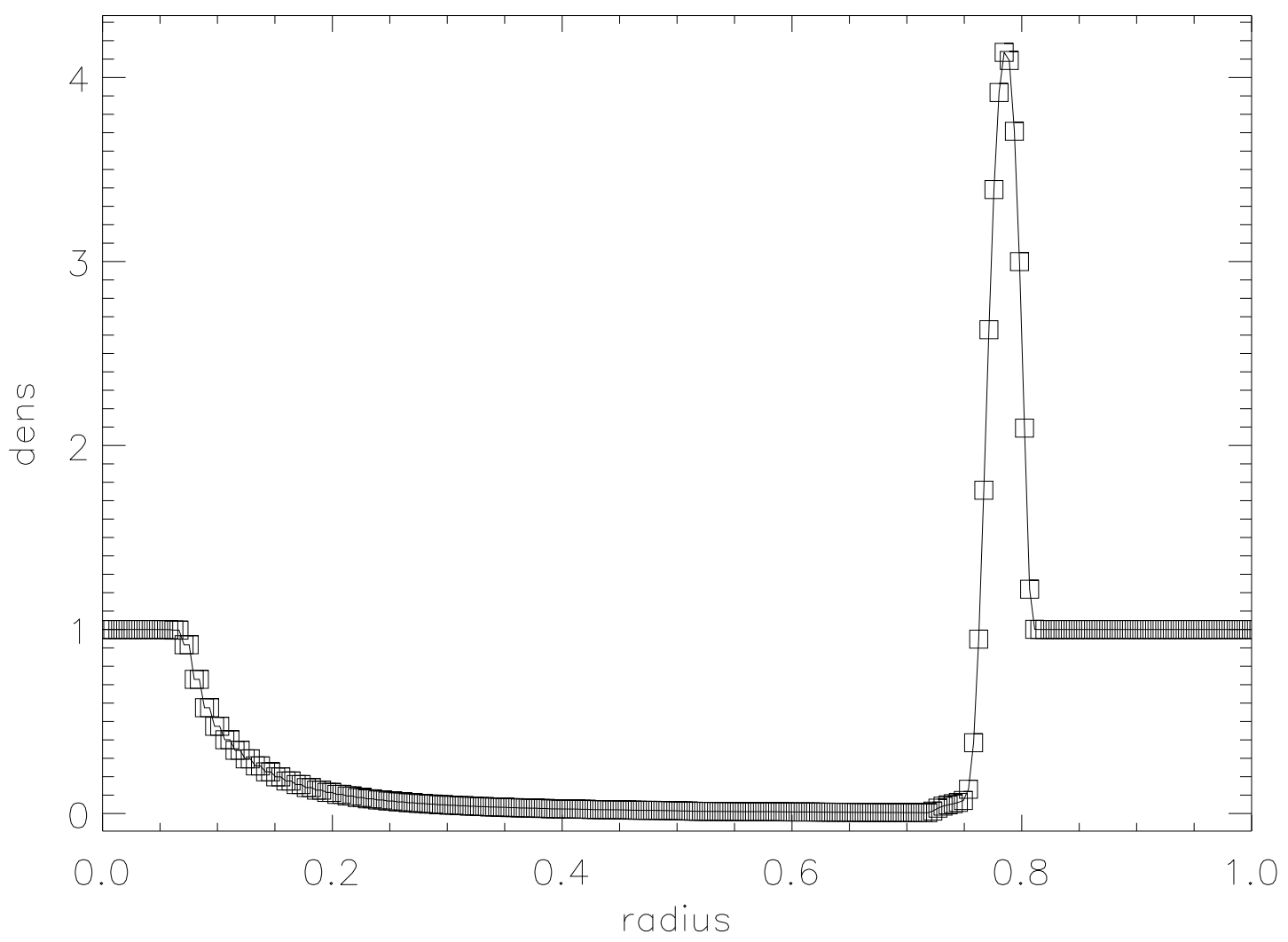

Fig. 31. - Spherical explosion in 2D cylindrical coordinates at $t=0.4$. Density along the $r=z$ line is plotted for results on an adaptive grid with a maximum resolution of $320 \times 320$ grid points(solid line and squares). The front of the shock is captured by $\sim 4$ points. 